ARTICLE

\title{
Epigenetic regulation of puberty via Zinc finger protein-mediated transcriptional repression
}

\author{
Alejandro Lomniczi ${ }^{1, \star}$, Hollis Wright ${ }^{1, \star}$, Juan Manuel Castellano ${ }^{1,2, \star \dagger} \dagger$, Valerie Matagne ${ }^{1, \star, \dagger}$, Carlos A. Toro ${ }^{1}$, \\ Suresh Ramaswamy ${ }^{3}$, Tony M. Plant ${ }^{3} \&$ Sergio R. Ojeda ${ }^{1}$
}

In primates, puberty is unleashed by increased GnRH release from the hypothalamus following an interval of juvenile quiescence. GWAS implicates Zinc finger (ZNF) genes in timing human puberty. Here we show that hypothalamic expression of several ZNFs decreased in agonadal male monkeys in association with the pubertal reactivation of gonadotropin secretion. Expression of two of these ZNFs, GATAD1 and ZNF573, also decreases in peripubertal female monkeys. However, only GATAD1 abundance increases when gonadotropin secretion is suppressed during late infancy. Targeted delivery of GATAD1 or ZNF573 to the rat hypothalamus delays puberty by impairing the transition of a transcriptional network from an immature repressive epigenetic configuration to one of activation. GATAD1 represses transcription of two key puberty-related genes, KISS1 and TAC3, directly, and reduces the activating histone mark $\mathrm{H} 3 \mathrm{~K} 4 \mathrm{me} 2$ at each promoter via recruitment of histone demethylase KDM1A. We conclude that GATAD1 epitomizes a subset of ZNFs involved in epigenetic repression of primate puberty.

\footnotetext{
${ }^{1}$ Division of Neuroscience, Oregon National Primate Research Center, Beaverton, Oregon 97006, USA. ${ }^{2}$ Department of Cell Biology, Physiology and Immunology, University of Cordoba; CIBER Fisiopatología de la Obesidad y Nutrición, Instituto de Salud Carlos III; and Instituto Maimónides de Investigación Biomédica (IMIBIC)/Hospital Universitario Reina Sofia (HURS), Cordoba 14004, Spain. ${ }^{3}$ Department of Obstetrics, Gynecology and Reproductive Sciences, University of Pittsburgh School of Medicine, Pittsburgh, Pennsylvania 15261, USA. * These authors contributed equally to this work. $\dagger$ Present addresses: Department of Cell Biology, Physiology and Immunology, University of Cordoba; or CIBER Fisiopatología de la Obesidad y Nutrición, Instituto de Salud Carlos III; or Instituto Maimónides de Investigación Biomédica (IMIBIC)/Hospital Universitario Reina Sofia (HURS), 14004 Cordoba, Spain (J.M.C.); Aix Marseille Université, INSERM, GMGF UMR_S 910, 13385, Marseille, France (V.M.). Correspondence and requests for materials should be addressed to T.M.P. (email: planttm@mwri.magee.edu) or to S.R.O. (email: ojedas@ohsu.edu).
} 
P uberty in highly evolved primates is unleashed after a prolonged phase of juvenile development by the reemergence of a robust pattern of pulsatile GnRH release, a mode of hypothalamic neuroendocrine activity that was placed in check during infancy ${ }^{1}$. One immediate cause of this juvenile hiatus in $\mathrm{GnRH}$ release appears to be a reduced production of the peptide kisspeptin from a subset of KISS1 neurons that are located in the arcuate nucleus (ARC) of the medial basal hypothalamus $(\mathrm{MBH})$. Because many of these KISS1 neurons also express neurokinin $\mathrm{B}$ and dynorphin, they have been termed $\mathrm{KNDy}$ neurons ${ }^{2,3}$. Both the reduced activity of the GnRH pulse generator during juvenile development and the resurgence of GnRH secretion at puberty occur in the absence of the gonads, indicating that these changes do not require feedback actions of either ovarian or testicular steroids in primates ${ }^{1}$.

One of our laboratories recently provided evidence that a mechanism of transcriptional repression prevents the premature initiation of puberty in the female rat by silencing the Kiss 1 gene in $\mathrm{KNDy}$ neurons ${ }^{4}$. Transcriptional repression is a major component of the molecular machinery regulating mammalian gene expression. Of the 2,000 transcription factors estimated to be present in the human genome ${ }^{5}$, almost 800 are transcriptional repressors containing zinc finger (ZNF) motifs ${ }^{6}$. These motifs recognize specific DNA sequences in regulatory regions of the genome $^{7}$, and therefore are critical for ZNF proteins to interact with DNA targets and regulate gene expression. The most common of these motifs is the $\mathrm{C} 2 \mathrm{H} 2$ or Krüppel type of ZNF $(\mathrm{KZNF})^{6}$. About one third of KZNFs also contain a motif called a Krüppel-associated box (KRAB) that recruits histone deacetylase complexes to the DNA region to which the ZNF is attached ${ }^{8}$.

The potential contribution of $Z N F$ genes to the pubertal process was recently suggested by several genome-wide association studies (GWAS) showing that single-nucleotide polymorphisms located near ZNF131, ZNF462 and ZNF483 were associated with an earlier age of menarche ${ }^{9-11}$. These observations raise the possibility that members of the ZNF gene superfamily, operating within the neuroendocrine brain, may represent a component of the neurobiological brake that underlies the arrest of the GnRH pulse generator during juvenile development in primates and thus contribute to preventing the premature initiation of puberty.

To systematically pursue this idea, we used DNA arrays to interrogate the hypothalamic transcriptome of agonadal male rhesus monkeys at two critical phases of postnatal development, the infantile-juvenile (INF-JUV) transition when the GnRH pulse generator is brought into check and the juvenile-pubertal (JUV-PUB) transition when robust GnRH pulse generator activity is re-initiated. We employed agonadal male monkeys to eliminate the confounding effect of gonadal steroids on gene expression in the brain, and because the juvenile hiatus in $\mathrm{GnRH}$ release is more conspicuous than that in females ${ }^{1}$. We also determined if any of the changes observed in males also occurred in females. To gain mechanistic insights into the actions of ZNFs, we used female rats as an in vivo model of puberty timing, and a combination of targeted DNA arrays, systems biology and in vitro molecular approaches. Our results suggest that ZNFs restrain puberty by epigenetically repressing a gene network that-operating in the ARC-controls puberty by governing pulsatile GnRH release.

\section{Results}

Gonadotropin levels in agonadal male monkeys. Consistent with earlier observations ${ }^{1}$, when monkeys were orchidectomized shortly after birth, serum luteinizing hormone (LH) and follicle a

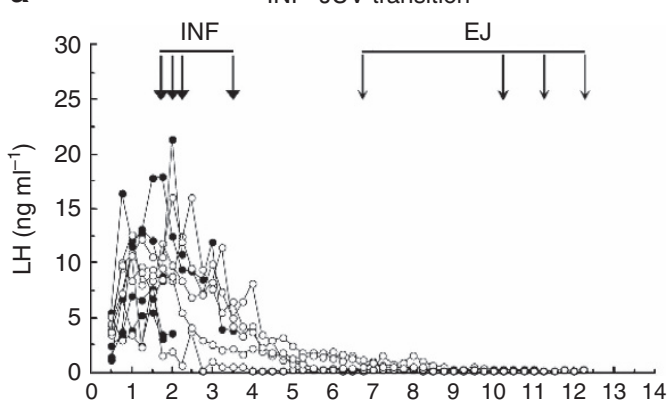

b

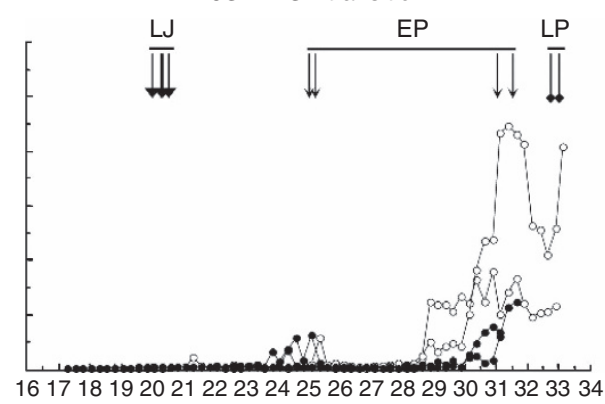

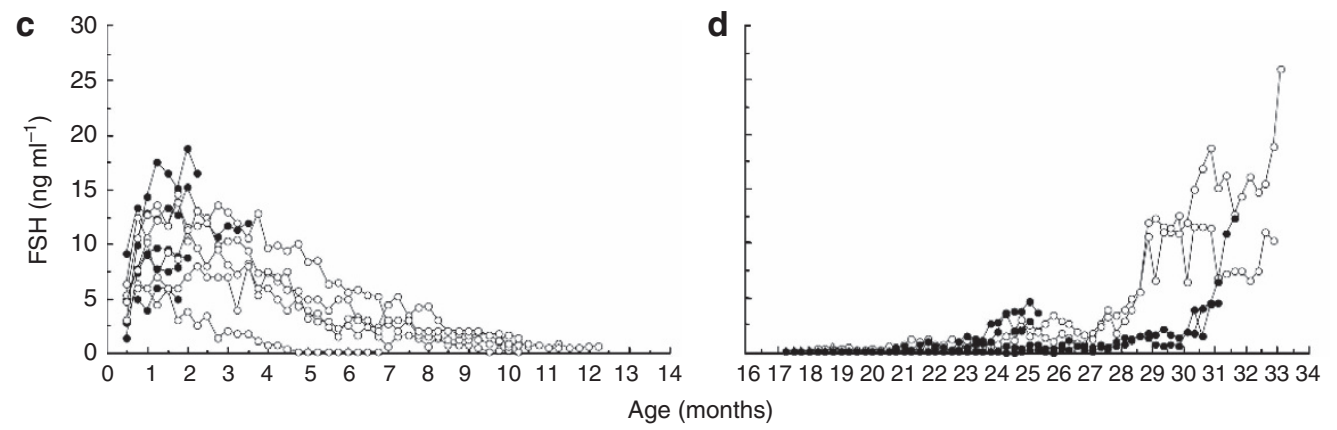

Figure 1 | Patterns of gonadotropin secretion in agonadal male monkeys. (a,c) Infant (INF)-juvenile (JUV) transition. Time courses of circulating concentrations of LH (a) and FSH (c) in 10 individual monkeys bilaterally orchidectomized at 2-6 days of age. Data for animals comprising the infantile (INF; $n=5)$ and early juvenile (EJ; $n=5)$ groups are shown by the closed and open data points, respectively. Block and line arrowheads indicate age at which the hypothalamus was collected from INF and EJ animals, respectively. (b,d) Juvenile (JUV)-pubertal (PUB) transition. Time courses of circulating concentrations of $\mathrm{LH}(\mathbf{b})$ and FSH (d) in 10 individual monkeys bilaterally orchidectomized at 16-18 months of age. Data for animals comprising the early pubertal (EP; $N=4)$ and late pubertal (LP; $n=2)$ groups are shown by the closed and open data points, respectively. Data for late juveniles (LJ) ( $n=4)$ are shown in open triangles-note these are largely masked by the closed data points. Block, line and square arrowheads indicate age at which the hypothalamus was collected from LJ, EP and LP animals, respectively. Thicker arrows are the result of two animals with overlapping ages. 
stimulating hormone (FSH) levels (reflecting pulsatile GnRH release) increased immediately and remained elevated for 3-4 months before decreasing to reach low, often undetectable, values by $9-12$ months of age (Fig. 1a,c). Similarly, when animals were orchidectomized at the mid-juvenile stage (16 months of age), serum LH and FSH levels remained low for at least 7-8 months, before increasing towards pubertal values (Fig. 1b,d; see also Supplementary Information).

ZNF expression during the JUV-PUB transition. Using Affymetrix GeneChip Rhesus macaque Genome Arrays to interrogate the $\mathrm{MBH}$ of agonadal male monkeys, we observed a significant $(P=0.0065$; hypergeometric test) enrichment in the category of transcriptional regulation within a group of 822 genes with decreased expression at the time of the pubertal rise in LH secretion (Supplementary Data 1). A conspicuous fraction of these genes belongs to the ZNF family of transcriptional repressors. Heat maps constructed using 161 ZNFs (Supplementary Data 2) with present calls in the array, revealed that $89 \mathrm{ZNF}$ belonging to the large KZNF gene cluster located on chromosome 19q (Fig. 2a; Supplementary Data 2), and 32 KZNFs located in chromosomes other than Ch19 (Supplementary Data 2), had a collective expression profile with the advent of the pubertal LH rise left-skewed towards decreased expression (Fig. 2b-e). This skewness was not observed in a set of 39 primate-specific ZNFs (Supplementary Data 2) mostly located in chromosomal region 19p12-p13.1 (Fig. 2a). Primate-specific genes were expressed in a symmetric, unimodal distribution with zero skewness (Fig. 2f,g), suggesting that they do not contribute to regulating the timing of puberty.

Based on these observations we selected $14 \mathrm{KZNF}$ for quantitative PCR (qPCR) analysis and found that expression of eight of them significantly decreased at the time of the pubertal gonadotropin rise (Fig. 3). Five of these genes (ZNF573, ZNF791, ZNF542/383, ZNF582 and ZNF587, listed in centromeric to telomeric order) are KRAB-KZNFs located on chromosomal region $19 \mathrm{q} 13$. However, not all KRAB-KZNFs showed a pubertyrelated expression profile, as the hypothalamic mRNA levels of ZNF483, a KRAB-KZNF located on a different chromosome (9q31.2-31.3) and implicated by GWAS in the timing of human menarche ${ }^{9,10}$, did not change at the time of the pubertal gonadotropin rise (Fig. 3).

Conversely, chromosome 19-located ZNFs were not the only ones exhibiting decreased expression in the hypothalamus at the time of the pubertal gonadotropin rise, as mRNA levels of three ZNFs lacking a KRAB domain and located in chromosomes other than chromosome 19 (ZNF207, ZNF462 and GATAD1) decreased a
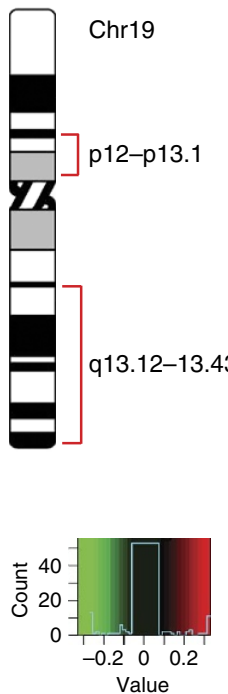

f Primate-specific KZNFs (Chr19p and off Chr19)

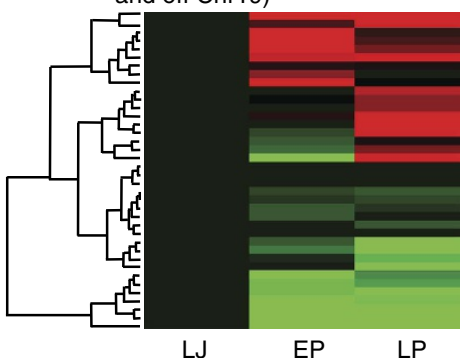

C
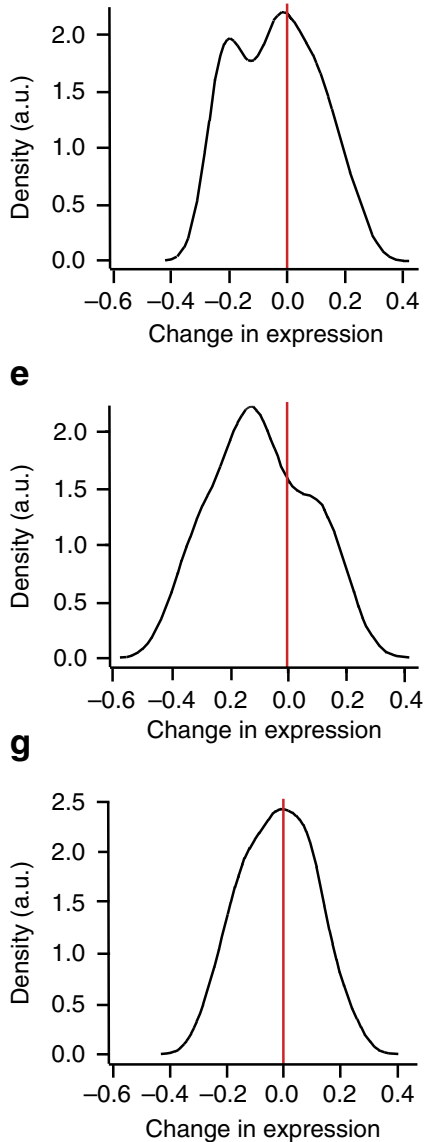

Figure 2 | Heat maps of ZNF expression in the MBH of agonadal monkeys. (a) Schematic representation of human chromosome 19 illustrating the location of two clusters of KZNF genes, the largest in the $q$ arm (q13.12-13.43 region) and another consisting of primate-specific genes in the $p$ arm (p12-p13.1 region). (b) Heat map illustrating the changes in expression of the KZNF cluster located in the $q$ arm during the juvenile-pubertal transition. (c) Kernel density plot of the changes shown in b. (d) Heat map illustrating the changes in expression of KZNFs located in chromosomes other than chromosome 19 during the juvenile-pubertal transition. (e) Kernel density plot of the changes shown in d. (f) Heat map of changes in expression of the primate-specific ZNF cluster located in either the $p$ arm of chromosome 19 or chromosomes other than chromosome 19. (g) Kernel density plot of the changes shown in f. LJ $(n=4), \operatorname{EP}(n=4), \operatorname{LP}(n=2)$. 

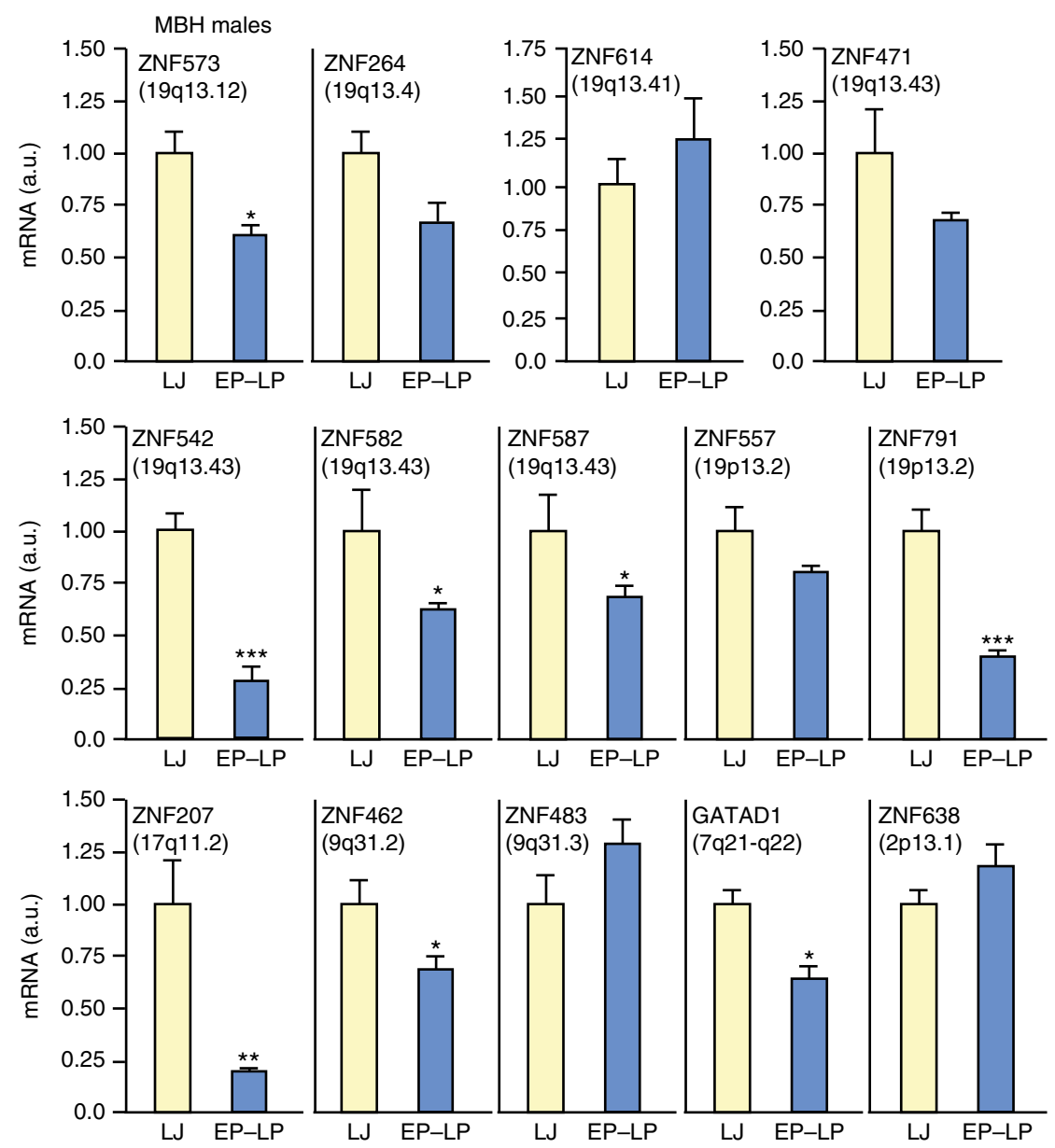

Figure 3 | ZNF mRNA levels in the monkey MBH at the juvenile-pubertal transition. During the juvenile-pubertal transition low levels of circulating gonadotropins begin to increase reflecting the reactivation of $\mathrm{GnRH}$ pulse generation that triggers puberty in intact monkeys (Fig. 1). All animals were orchidectomized at 16-18 months of age. Because mRNA levels did not change further in LP as compared with EP these two groups were combined before statistical analysis. Vertical bars are means \pm s.e.m. $\mathrm{LJ}=$ late juvenile $(n=4-6) ; \mathrm{EP}=$ early puberty and $\mathrm{LP}=$ late puberty $\left(n=5-8 ;{ }^{\star} P<0.05,{ }^{\star \star} P<0.01\right.$ and ${ }^{\star \star \star} P<0.001$ versus LJ group; Student's t-test).

at this time (Fig. 3). ZNF462 is located in the same chromosomal region as ZNF483 and has also been implicated by GWAS as affecting the timing of menarche in girls ${ }^{9,10}$. GATAD1 (GATA ZNF domain-containing protein 1 , also known as $O D A G$, ocular development-associated gene) contains a ZNF domain at the $\mathrm{N}$ terminus $^{12}$, and is located on chromosomal region $7 \mathrm{q} 21-\mathrm{q} 22$ (ref. 13). GWAS have implicated GATAD1 as a gene associated with adult height ${ }^{14,15}$. GATAD1 is considered as a histone 'reader' that recognizes promoter regions containing histone 3 trimethylated at lysine $4(\mathrm{H} 3 \mathrm{~K} 4 \mathrm{me} 3)$ to form a repressive complex ${ }^{16}$.

Of the eight ZNFs with decreased pubertal expression in the $\mathrm{MBH}$ of agonadal male monkeys, expression of five of them also decreased in the cerebral cortex (CTX) (Supplementary Fig. 1) suggesting that this developmental decrease was not hypothalamic specific. In contrast, mRNA levels of three ZNFs (ZNF573, ZNF542 and GATAD1) remained unchanged in the CTX (Supplementary Fig. 1). To determine whether peripubertal repression of these three genes was associated with changes in expression of other genes previously implicated in the control of puberty (for example, KISS1, TAC3, GnIH and so on), we further mined the DNA array results and observed that expression of only KISS1 and TAC3 appeared to increase at the time when ZNF573, ZNF542 and GATAD1 expression decreased (Supplementary Data 3). QPCR analysis demonstrated that indeed both KISS1 and TAC3 mRNA content increased significantly in the $\mathrm{MBH}$ at this time (Supplementary Fig. 2). Of the three ZNFs showing a hypothalamic-specific decreased expression in males, only GATAD1 and ZNF573 behaved similarly in intact female monkeys of pubertal age (Supplementary Fig. 3), suggesting that these genes may be involved in the pubertal process of both sexes.

ZNF expression during the INF-JUV transition. Of the eight ZNFs with decreased expression at the time of the pubertal reactivation of gonadotropin release in agonadal males, only GATAD1 expression increased in the $\mathrm{MBH}$ at the time of the INF-JUV transition (Fig. 4). This finding suggests that GATAD1 may play a dual role in both silencing the $\mathrm{GnRH}$ pulse generator at the INF-JUV transition and the awakening of GnRH release at the onset of puberty.

To determine whether upregulation of GATAD1 expression at this stage of postnatal development could be related to changes in expression of genes thought to be involved in the reactivation of GnRH pulse generator activity at the onset of puberty, we examined the DNA array results for a number of these genes including those associated with initiation (for example, KISS1, TAC3 and so on), and delay (for example, GnIH, genes encoding opioid peptides, GABA-related peptides and so on) of puberty. None exhibited significant changes in the expression 

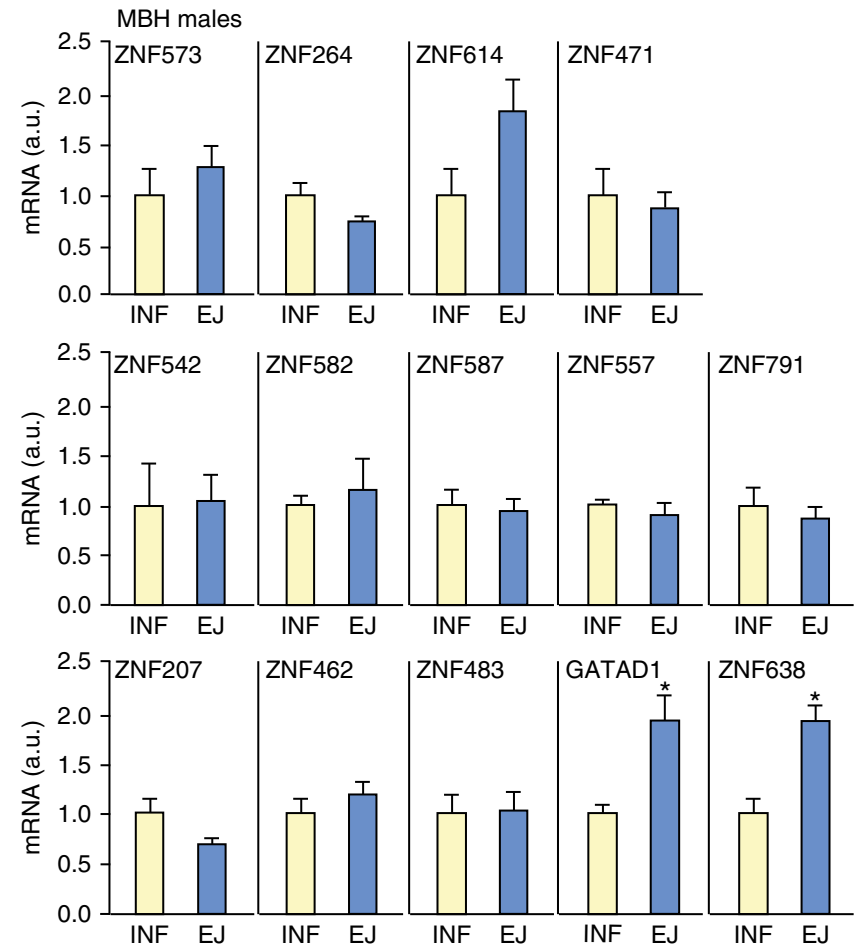

Figure 4 | ZNF mRNA levels in the monkey MBH at the infantile-early juvenile transition. During the infantile-early juvenile transition, the elevated gonadotropin levels seen in infancy (reflecting a robust $\mathrm{GnRH}$ pulsatility) are brought into check resulting in the hypogonadotropic state of the juvenile phase of development. All animals were orchidectomized 2-6 days after birth. Vertical bars are means \pm s.e.m. INF $=$ infant $(n=3-5)$; $\mathrm{EJ}=$ early juvenile $(n=4-5) .\left({ }^{*}=P<0.05\right.$ versus INF group; Student's $t$-test).

(Supplementary Data 4, Supplementary Fig. 2), indicating the need for additional research to unveil the mechanisms underlying $\mathrm{GnRH}$ pulse generation by the infant hypothalamus.

GATAD1 and ZNF573 delay puberty in a rodent model. To determine whether modifying GATAD1 or ZNF573 expression alters the timing of puberty, we chose a gain-of-function approach. We cloned the coding region of hGATAD1 tagged with the human influenza haematogglutinin epitope (HA) or human ZNF573-tagged with both MYC and FLAG epitopes into a lentivirus vector (LV) that expressed GFP (Supplementary Fig. 4a). Both constructs (termed LV-GATAD1 and $L V$-ZNF573) express the proteins of interest in 293T cells (Supplementary Fig. 4b). Although endogenous Gatad1 mRNA levels do not decrease in the $\mathrm{MBH}$ of female rats during prepubertal development (Supplementary Fig. 5) and Znf573 has not been described in rats, we nevertheless used the immature female rat as an in vivo system to broadly test the concept that ZNFs can affect the timing of puberty. We delivered these constructs bilaterally to the ARC via stereotaxic surgery into 21-day-old female rats. Female rats are a much better model than male rats to study puberty in rodents because the timing and completion of puberty can be precisely defined by detecting both the age at vaginal opening and first ovulation. The latter is determined indirectly by the presence of leukocytes in vaginal lavages the day after the animals were in the oestrus phase of the reproductive cycle (for additional details see Supplementary Information). Control animals were injected with a construct expressing only green fluorescent protein (GFP; LV-GFP). Immunohistofluorescence analysis of the sites of injection at the time of euthanasia (3 weeks after vaginal opening) using an antiGFP antibody demonstrated that the injections of LV-GATAD1 were located lateral and rostral to the ARC in three animals (Fig. 5a), dorsal to the ARC in three others (Fig. 5b), and well-placed in the body of this nucleus in six rats (Fig. 5c,d). Both the age at vaginal opening and the age at first ovulation were delayed (Fig. 5e,f), and oestrous cyclicity was disrupted (Fig. 5g). $L V$-GATAD1-injected rats spent fewer days in proestrous and more days in diestrous than control animals (Fig. 5h). Although ovarian weight (measured at the time of euthanasia) was lower in LV-GATAD1-injected animals, this difference did not reach statistical significance (Fig. 5i). Similar changes were observed in animals injected with $L V$-ZNF573 (Supplementary Fig. 6). Eight days after $L V$-GATAD1 injection into the ARC $(n=8)$, hGATAD1 mRNA levels were readily measurable, in contrast to LV-control-injected animals $(n=8)$ in which hGATAD1 transcripts were undetectable (Fig. $5 \mathrm{j}$ ). The presence of hGATAD1 mRNA was accompanied by a significant decrease in both Kiss 1 and Tac3 mRNA abundance (Fig. 5j). These results suggest that a subgroup of ZNFs, epitomized by GATAD1 and ZNF573, operates within the mammalian hypothalamus to prevent the premature activation of gonadal function.

GATAD1 disrupts prepubertal reorganization of gene networks. To determine whether overexpressing GATAD1 in the ARC alters the connectivity of genes deemed to be important for pubertal development, we assembled a set of 224 genes (Supplementary Data 5) involved in diverse cellular functions, including trans-synaptic communication, glia-to-neuron communication, cell adhesiveness, transcriptional control and epigenetic regulation (Supplementary Fig. 7). We then generated custom-made TaqMan Open Array plates (Applied Biosystems) to interrogate the ARC transcriptome at three phases of prepubertal development (infantile-PND14, early juvenile (EJ)-PND21 and late juvenile (LJ)-PND28). The latter corresponds to the end of the juvenile period. We also examined the ARC 7 days after targeting GATAD1 to this region in PND21 rats. The TaqMan Open Array reliably detected 147 of the 224 genes (Supplementary Data 6). Failure to detect all 224 transcripts may be due to low amplification efficiency of computationally selected primers and to the low amount of reverse-transcribed material used in each microreaction $(33 \mathrm{nl})$, which makes less certain the detection of low-abundance transcripts. To analyse the network configuration and connectivity of these genes, we used a compressive-sensing-based approach ${ }^{17}$, which infers gene networks by identifying strong co-expression links between genes and uses these links to estimate the potential influence of each of these genes within a given transcriptional programme (Supplementary Information).

Co-expression networks inferred using this method showed that the genes losing the most cumulative connectivity (edges) over time have considerable connectivity among themselves at PND14 (Fig. 6a, green circles), but they are also connected to gene sets that ultimately gain connectivity (Fig. 6a, red circles). By PND21, these relationships begin to shift (Fig. 6b), and by the JUV-PUB transition (PND28), the genes losing edges are almost entirely disconnected from each other (Fig. 6c, green circles), whereas genes gaining edges become more interconnected (Fig. 6c, red circles).

Overexpressing GATAD1 in the ARC at PND21 resulted in an immature pattern of connectivity at PND28 resembling that of PND14. The top 20 genes losing edges under GATAD1 overexpression, are normally richly interconnected at PND28 (Fig. 6d, yellow circles, triangles, squares; for definition of these shapes see legend to Fig. 6). Conversely, the top 20 genes gaining 
a

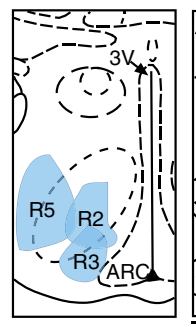

Bregma $-2.3 \mathrm{~mm}$ b
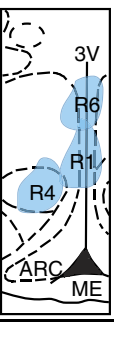

C

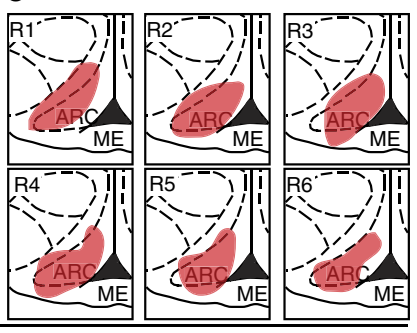

d

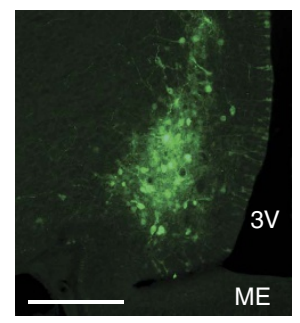

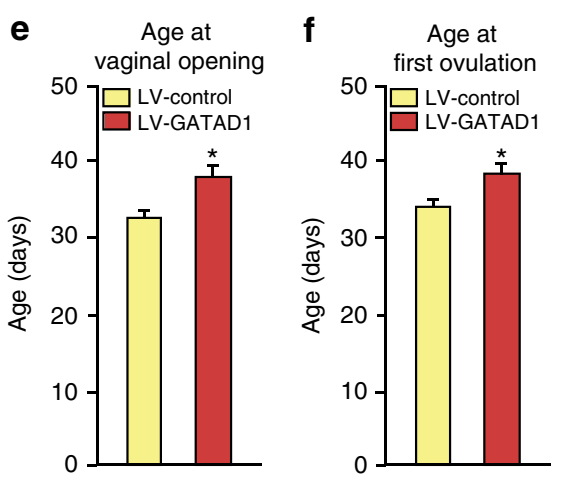

g
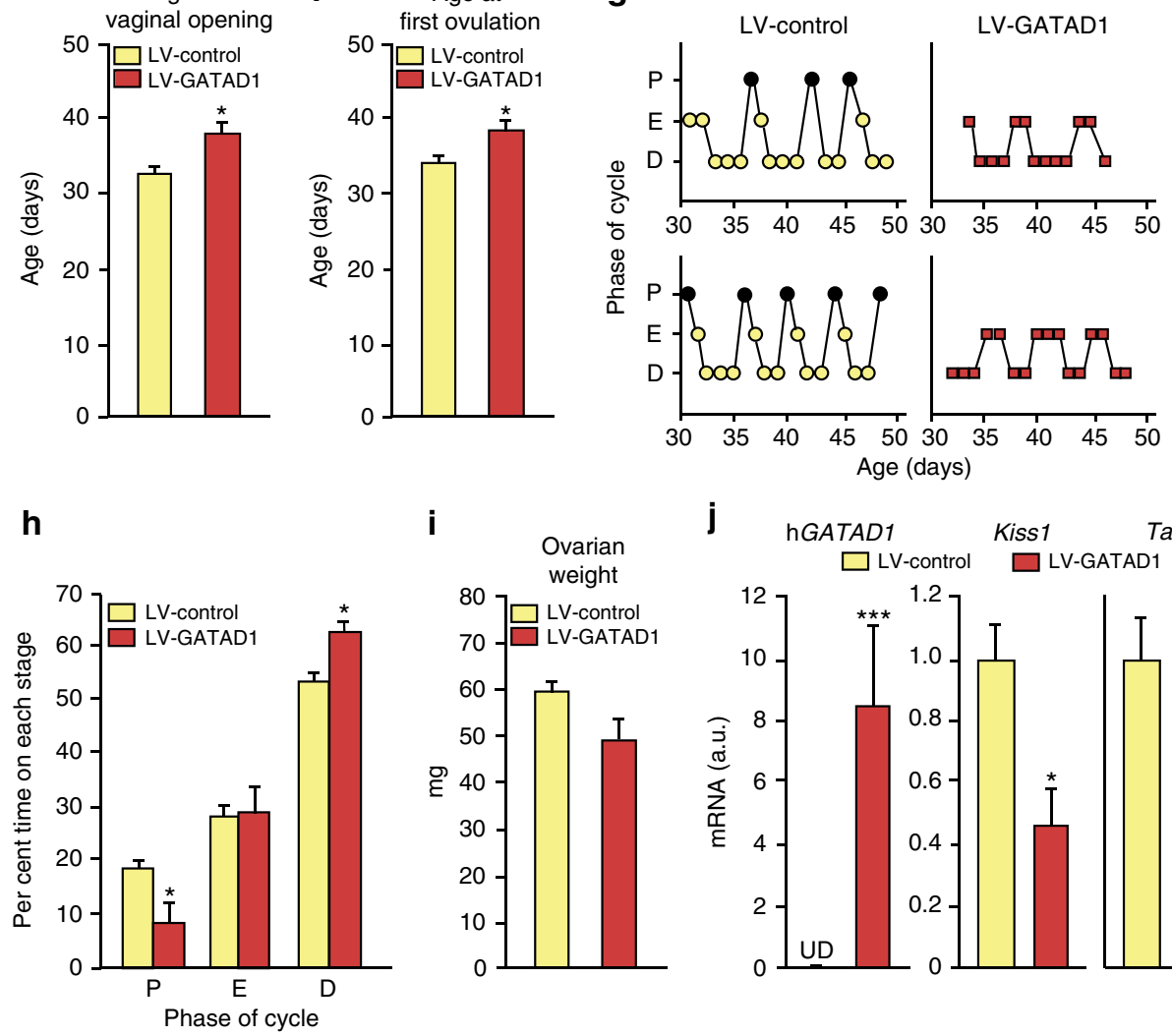

i
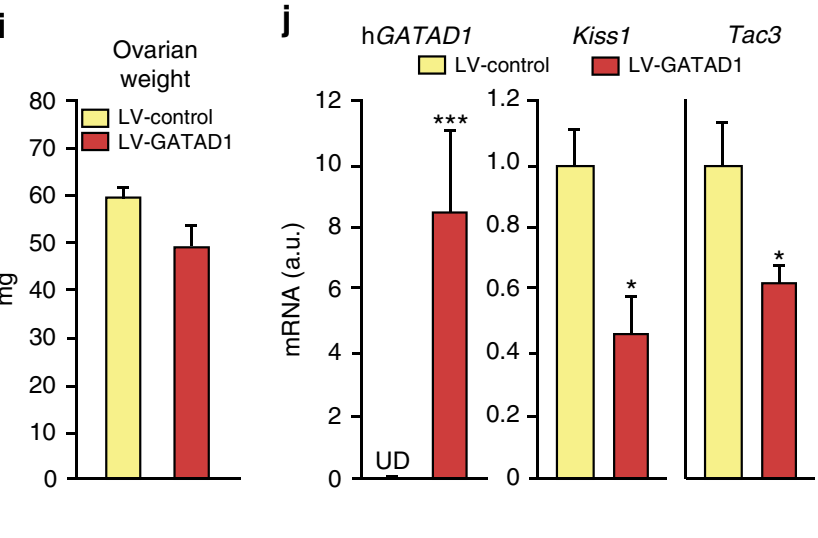

Figure 5 | Overexpression of hGATAD1 in the ARC of immature female rats. (a-c) Diagrams showing the approximate location of microinjections intended to deliver a lentivirus construct expressing hGATAD1 (LV-GATAD1) to the ARC nucleus of prepubertal female rats. (a) Injections located anterior and lateral to the intended target $(n=3) ;(\mathbf{b})$ injections located dorsal to the intended target $(n=3) ;(\mathbf{c})$ injections correctly located in the body of the ARC $(n=6)$. (d) Immunohistofluorescent localization of GFP-expressing cells in the ARC of a female rat 30 days after receiving injections of LV-GATAD1. The construct was stereotaxically delivered to the ARC on PND21. One coronal plane, showing the right side of the MBH is depicted. Bar, $200 \mu \mathrm{m}$. Because rats injected with an LV-GFP construct or rats with misplaced LV-GATAD1 injections (too high or too rostral/lateral) behave similarly, they were combined into a single group (LV-control) for statistical purposes. (e) The age at vaginal opening was delayed in LV-GATAD1-injected rats. ( ${ }^{\star} P<0.05$ versus LV-control group; Student's t-Test) (f) The age of first ovulation, estimated by a predominance of leukocytes in vaginal lavages following an abundance of cornified cells (indicative of the first oestrus) was also delayed. ( ${ }^{\star} P<0.05$ versus LV-control group; Student's $t$-test; $\mathbf{g}$ ) Examples of disrupted oestrous cyclicity in LV-GATAD1-injected rats. (h) Per cent of time spent in different stages of the oestrous cycles by rats injected with LV-GATAD1 in the ARC (LV-GATAD1) versus control animals (LV-control). ( ${ }^{\star} P<0.05$ versus LV-control group; Student's $t$-test) (i) Ovarian weight in LV-GFP and LV-GATAD1-injected rats.

(j) $h$ GATAD1, Kiss1 and Tac3 mRNA levels detected by qPCR in the ARC of 28-day-old female rats receiving an LV-GATAD1 ( $n=7$ ) injection targeting the ARC on PND21. ( ${ }^{\star} P<0.05$ and ${ }^{\star \star \star} P<0.001$ versus LV-control group $(n=8)$; Student's $t$-test). Vertical bars are means \pm s.e.m. ME $=$ median eminence; $3 \mathrm{~V}=$ third ventricle. $\mathrm{P}=$ proestrous; $\mathrm{E}=$ oestrous; $\mathrm{D}=$ diestrous.

connectivity are poorly connected at PND28 (Fig. 6, blue circles, triangles, squares).

Both the top 20 losers and gainers of connectivity during prepubertal development were enriched for a number of functional annotations according to the DAVID functional enrichment analysis tool (Supplementary Data 7 and 8). Some of these functions would be expected to be important for the regulation of the pubertal process, because genes gaining edges between PND14 and PND28 are likely to have increased influence on the transcriptional programme of ARC cells as puberty approaches, while genes losing edges during the same time interval may become less influential. To determine whether GATAD1 overexpression changes the connectivity of these functionally annotated genes, we performed a computational analysis of data derived from two relevant functional enrichment categories, GO, using the category 'negative regulation of transcription, DNA-templated', and the Protein Information Resource using the keyword 'transcription regulation'. The results 
a

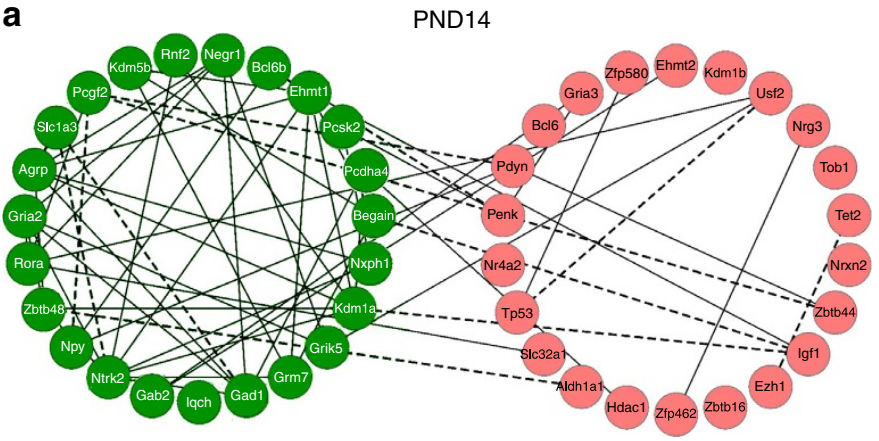

e GO:0045892

b

PND21

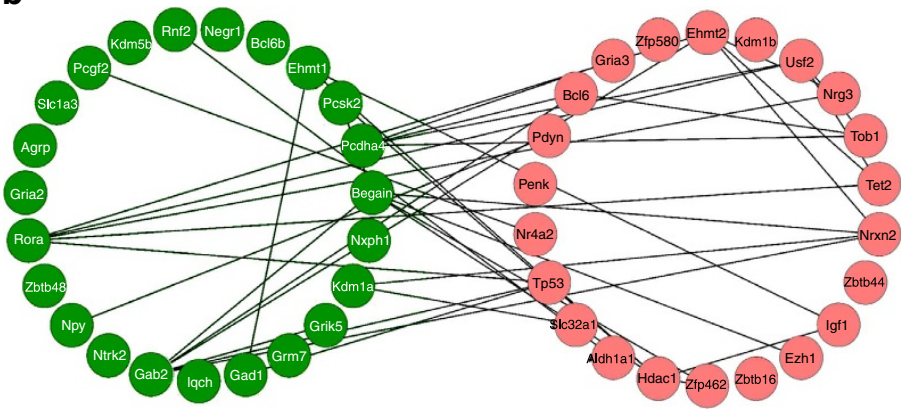

PIR

Negative regulation

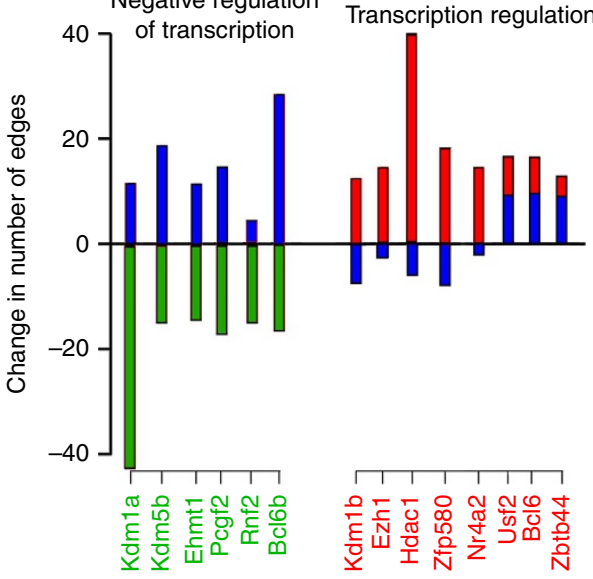

c

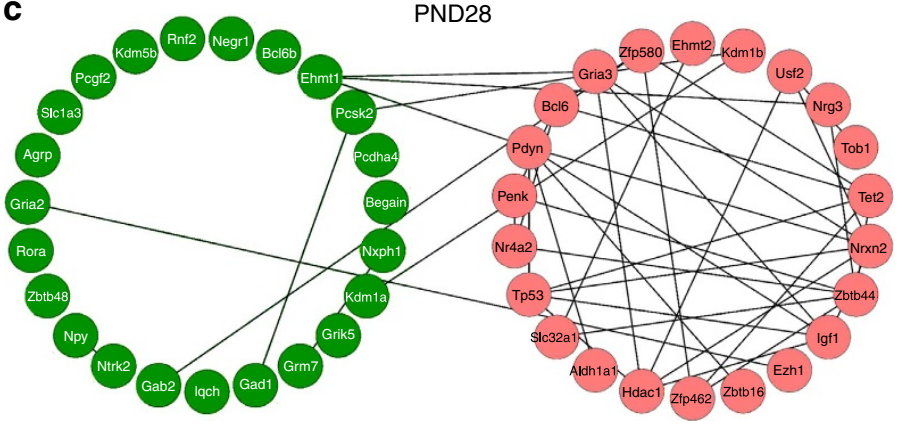

d

PND28 GATAD1 TOP 20
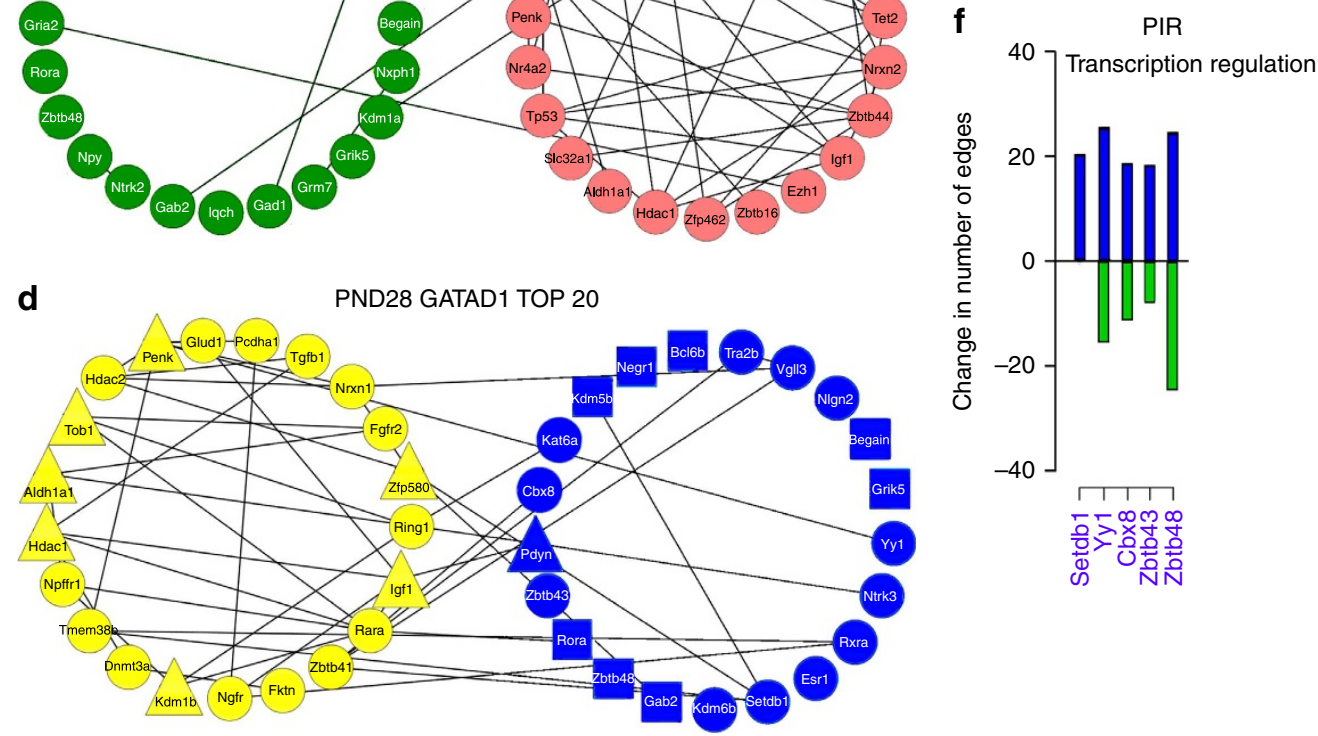

Figure 6 | Effect of GATAD1 overexpression on gene co-expression networks. (a-c) Inferred co-expression network between the top 20 genes losing edges (green) and the top 20 genes gaining edges/connections (red) during prepubertal development, based on sum of changes in connectivity between PND14-PND21, PND14-PND28 and PND21-PND28. (a) Network at PND14. (b) Network at PND21. (c) Network at PND28. (d) Network at PND 28 with the top 20 genes losing edges (yellow) and gaining edges (blue) under GATAD1 overexpression as compared with the network inferred at PND28. In this network, genes that are among the top 20 genes gaining edges during normal prepubertal development are shown as triangles, while genes that are among the top 20 losers of connectivity during development are shown as squares. For all networks positive correlation edges are represented by solid black lines, negative correlation edges are shown as dashed lines. (e) Changes in connectivity of genes belonging to selected transcription-related functional categories that lose (Gene Ontology category GO:0045892: negative regulation of transcription, DNA-templated) or gain (Protein Information Resource,

http://pir.georgetown.edu/; Keyword 'transcription regulation') connectivity (green and red bars, respectively) from PND14 to PND28. For each gene the alteration in connectivity resulting from GATAD1 overexpresssion as compared with PND28 is shown as blue bars. (f) Genes encoding transcriptional repressors (annotated by 'SP_PIR_KEYWORD' 'transcription regulation') belong to the group of top 20 gainers of connectivity under GATAD1 overexpression (blue bars). Most of these genes (except Setdb1) showed decreased connectivity during normal prepubertal development (green bars). 
showed that epigenetic repressors, such as the histone demethylases Kdm1a and Kdm5b, and the PcG-related genes Ehmt1, Pcgf2 and Rnf2 lose connectivity as puberty approaches in the female rat (Fig. 6e, green bars), and that GATAD1 overexpression reverses this change (Fig. 6e, blue bars). We also identified at least three epigenetic regulators $(K d m 1 b, E z h 1$ and $H d a c 1)$, and one transcriptional repressor ( $Z f p 580)$ among the top 20 genes gaining connectivity over normal development (Fig. 6e, red bars), but that fail to do so under GATAD1 overexpression (Fig. 6e, blue bars). Interestingly, several of the top 20 gainers of connectivity under GATAD1 overexpression (Fig. 6f, blue bars) were repressive genes, which normally either lose $(Y y 1, C b x 8, Z b t b 43$ and $Z b t b 48)$ or maintain the same number of edges (Setdb1) between PND14 and PND28 (Fig. 6f, green bars). Setdb1, encodes an enzyme that trimethylates histone 3 at lysine 9 (ref. 18) (and therefore generates a repressive histone mark), $Y y 1$ and $C b x 8$ are members of the PcG silencing complex, and Zbtb43 and Zbtb48 are transcriptional repressors of the POZ-BTB family. Overall, these results strongly suggest that genes involved in epigenetic repression that lose influence in gene co-expression networks as puberty approaches regain this influence under GATAD1 overexpression.

GATAD1 represses KISS1 and TAC3 transcription. Promoter assays using constructs carrying the proximal promoter of several genes, including the puberty-activating genes $h K I S S 1, h T A C 3$ and $r T t f 1$, and the puberty repressive genes $r E e d, h E A P 1, r p E n k$ and rViaat demonstrated that GATAD1 only repressed the promoter activity of $h K I S S 1$ and $h T A C 3$ (Fig. 7a,b), without affecting the promoter activity of any of the other genes (Fig. 7c-g).

Chromatin immunoprecipitation (ChIP) assays showed strong association of GATAD1 to the proximal promoter of both hKISS1 and hTAC3 (Fig. 7h,i). Consistent with the results of promoter assays, no significant GATAD1 association to two other promoters tested, $h E E D$ and $h E A P 1$, was detected (Fig. $7 \mathrm{j}, \mathrm{k}$ ).

The network analysis of the ARC showed that connectivity of $K d m 1 a / L s d 1$ decreases immediately before puberty, and that this decrease was prevented by GATAD1 overexpression. Because KDM1A, the product of the Kdm1a/Lsd1 gene, selectively demethylates $\mathrm{H} 3 \mathrm{~K} 4 \mathrm{me} 2$ at gene promoters ${ }^{19}{ }, \mathrm{Kdm} 1 \mathrm{a} / \mathrm{Lsd} 1$ is considered to be a gene involved in transcriptional repression $^{19,20}$. Consistent with this notion, KDM1A/LSD1 recruitment to the KISS1 and TAC3 promoters increased markedly after GATAD1 overexpression (Fig. 7l,m), without changing at the EED or EAP1 promoters (Fig. 7n,o). The increased KDM1A recruitment to the KISS1 and TAC3 promoters coincided with loss of $\mathrm{H} 3 \mathrm{~K} 4 \mathrm{me} 2$ (Fig. 7p), without changes in H3K4me3 abundance (Fig. 7q).

To determine whether the changes in GATAD1 association to the Kiss 1 and Tac3 promoters observed in cultured cells also occur in vivo during the normal initiation of primate puberty, we performed ChIP assays using chromatin derived from the hypothalamus of female rhesus macaques at the LJ and EP stages of pubertal development. The results showed that-consistent with the ChIP results in 293T cells (Fig. 7) -the association of GATAD1 and KDM1A to the KISS1 and TAC3 promoters also decreases in vivo (Fig. 8a,b), at the time when hypothalamic GATAD1 mRNA abundance declines (Fig. 3), and coinciding with an increase in the content of the activating histone mark H3K4me2 (Fig. 8a,b). We observed no change in GATAD1, KDM1A or H3K4me2 association to the EED promoter (Fig. 8c). Although there was some loss of GATAD1 from the EAP1 promoter at EP, this change was not accompanied by changes in either KDM1A or H3K3me2 association (Fig. 8d). These results suggest that GATAD1 delays puberty by repressing transcription of KISS1 and TAC3 in the ARC, both directly and by reducing the levels of the activating histone mark $\mathrm{H} 3 \mathrm{~K} 4 \mathrm{me} 2$ at each promoter, via recruitment of KDM1A/LSD1.

To determine whether Gatad1 transcripts are present in Kiss1-containing neurons of the ARC we utilized a double fluorescent in situ hybridization technique. We used late juvenile female rats for this study, because we had no available tissue from non-human primates. In addition to kisspeptin neurons, we examined two hypothalamic populations of neurons involved in the control of puberty, GABAergic and glutamatergic neurons. GABAergic neurons contribute to the inhibitory control of $\mathrm{GnRH}$ secretion and were visualized by detecting GAD67 mRNA. Glutamatergic neurons are involved in the excitatory control of $\mathrm{GnRH}$ and were visualized by detecting Nell2 $\mathrm{mRNA}^{21}$. As predicted by its transcriptional regulatory functions, Gatad 1 mRNA is expressed in cells throughout the $\mathrm{MBH}$, prominently in the ARC (Fig. 8e). Importantly, Gatad1 transcripts are present in all three neuronal populations studied (Fig. $8 \mathrm{f}-\mathrm{h}$ ), indicating that the transcriptional regulatory activity of GATAD1 on different genes is controlled by cell-specific factors.

GATAD1 and ZNF573 are connected to menarche genes. To determine whether GATAD1 and/or ZNF573 are functionally connected to genes implicated in the timing of human puberty, we used the GeneMANIA literature interaction database ${ }^{22}$ to analyse the known relationships that exist between ZNF573 and GATAD1, and more than 120 genes associated to the age of menarche ${ }^{11}$. When queried, GeneMANIA searches a host of databases describing known gene interactions such as co-expression, shared protein domains, genetic interactions, predicted interactions and shared biochemical and genetic pathways. We observed that this menarche-related gene cohort $^{11}$ is not only densely interconnected, but also connected to GATAD1 and ZNF573 (Supplementary Fig. 8a). GATAD1 and ZNF573 are significantly $(P=0.007$ and $P=0.0$; permutation test, effective $P$ value based on 1,000 replicates) more connected (Supplementary Fig. 8b) to their first neighbours within the overall menarche-related gene network than to 1,000 randomly networks of the same size containing randomly selected gene sets unrelated to menarche, extracted from the GeneMANIA database (Supplementary Fig. 8c). Representation of these connectivity distributions demonstrated that the degree of connectivity of both GATAD1 and ZNF573 to menarche-related genes (Supplementary Fig. 8d,e, single red line) was greater than the connectivity observed in random gene set-derived sub-networks (29 vs13.47 and 76 vs14.7, respectively; Supplementary Fig. 8d,e, black lines). It thus appears that GATAD1 and ZNF573 are functionally connected to a large cohort of genes that associate with an earlier age of menarche in girls.

\section{Discussion}

The present study provides evidence that members of the ZNF superfamily of transcriptional repressors represent an important component of the neurobiological brake that holds the $\mathrm{GnRH}$ pulse generator in check during juvenile development in highly evolved primates, and that, therefore, prevents the premature awakening of the pubertal process in these species. We show that expression of GATAD1 and ZNF573, along with that of five other ZNFs, decreases in the $\mathrm{MBH}$ of agonadal male rhesus monkeys during the JUV-PUB transition when GnRH pulse generator activity increases. Similar peripubertal changes in GATAD1 and ZNF573 expression were also observed in ovary-intact female monkeys. Moreover, when GATAD1 or ZNF573 were overexpressed in the ARC of immature female rats, puberty was delayed and subsequent oestrous cyclicity was disrupted. 

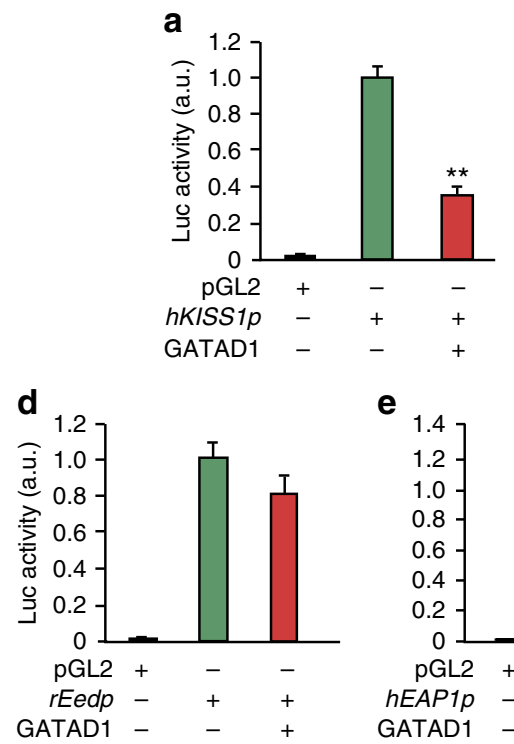

b

e

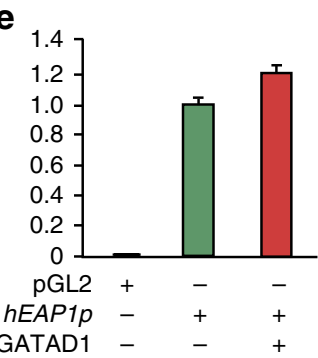

h

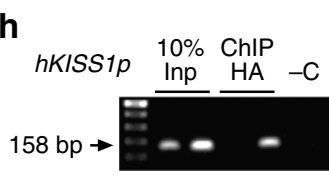

GATAD

$-+-+$

I

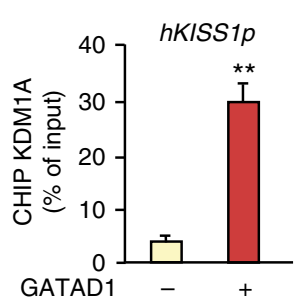

i

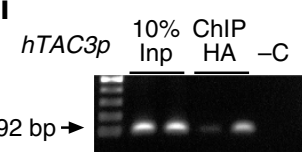

GATAD1 - + + +

m

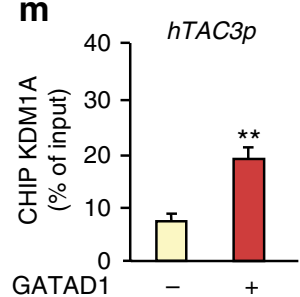

p

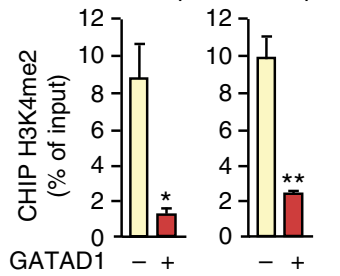

c
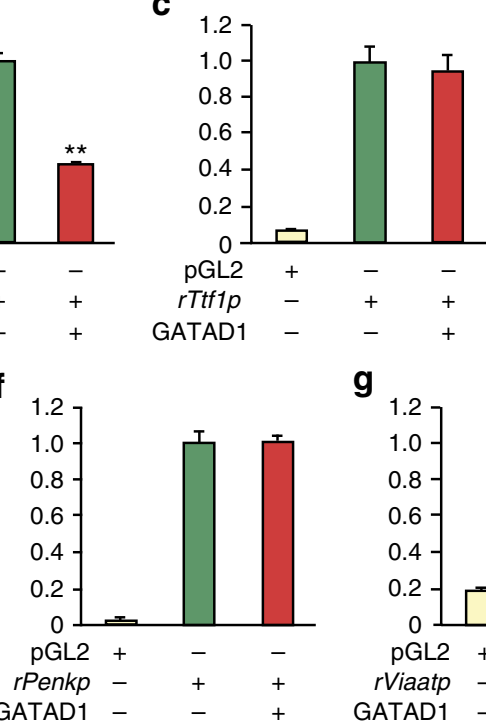

g

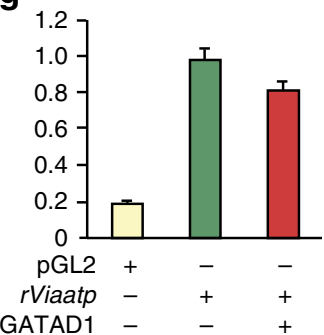

k

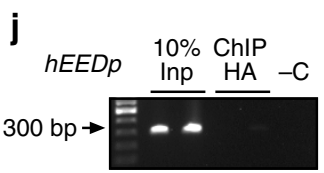

GATAD1 $-+\cdots$

n
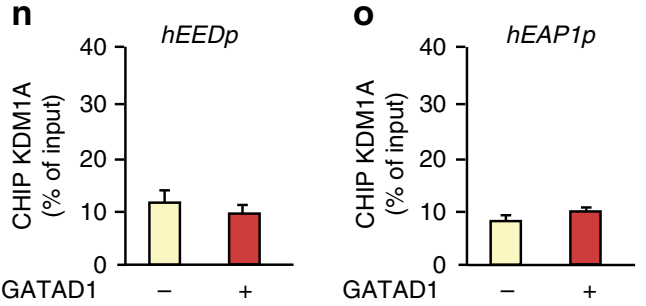

Figure 7 | GATAD1 is a transcriptional repressor of the KISS1 and TAC3 genes. (a) GATAD1 represses hKISS1 promoter activity as measured by gene reporter assays performed in Neuro2A cells; (b) GATAD1 also represses hTAC3 promoter activity (c-g) GATAD1 does not alter the transcriptional activity of other puberty-related genes; (c) rTff1, a transcriptional activator; (d) rEed, a transcriptional repressor; (e) hEAP1, a transcriptional regulator with dual repressive/activating capabilities; (f) rPenk, a gene encoding encephalin a neuropeptide that inhibits GnRH secretion; (g) rViaat, a gene encoding a vesicular inhibitory amino acid transporter, also considered to be inhibitory of the pubertal process $\left({ }^{\star \star} P<0.01\right.$ versus GATAD1 $(-)$ group $(n=5)$;

One-way ANOVA, Student-Newman-Keuls post hoc test). GATAD1 is recruited to (h) the hKISS1 promoter, and (i) the hTAC3 promoter, as assessed by ChIP assays performed in 293T cells; GATAD1 is not recruited to either ( $\mathbf{j}$ ) the hEED promoter, or ( $\mathbf{k})$ the hEAP1 promoter; GATAD1 enhances recruitment of KDM1A/LSD1 to both (I) the hKISS1 promoter, and $(\mathbf{m})$ the hTAC3 promoter; but not to the $(\mathbf{n})$ hEED promoter or $(\mathbf{o})$ hEAP1 promoter. GATAD1 depletes H3K4me2 from the (p) hKISS1 promoter and hTAC3 promoter, without changing the abundance of $(\mathbf{q})$ H3K4me3 $\left({ }^{\star} P<0.05\right.$ and ${ }^{\star \star} P<0.01$ versus GATAD1 $(-)$ group $(n=3)$; Student's $t$-test).

Our results show that four of the eight ZNFs displaying decreased expression in the male monkey hypothalamus at puberty (ZNF573, ZNF582, ZNF587 and ZNF791) belong to the large family of $K R A B-K Z N F$ genes. These four 'puberty-related' KRAB-KZNFs are members of the large cluster of KZNFs located in the q13 region of chromosome 19. It is unlikely, however, that all KRAB-KZNFs contribute to silencing genes that at the termination of juvenile development reactivate $\mathrm{GnRH}$ pulse generation, as evidenced by the finding that expression of ZNF483, a KRAB-KZNF located on chromosome 9, did not decrease during the JUV-PUB transition. Conversely, ZNFs lacking a KRAB domain and located in chromosomes other than chromosome 19 may work in concert with chromosome 19 KRAB-KZNFs to maintain puberty in check. In keeping with this idea, hypothalamic expression of GATAD1 and ZNF462, two ZNFs encoding proteins lacking a KRAB domain, decreased at puberty. 


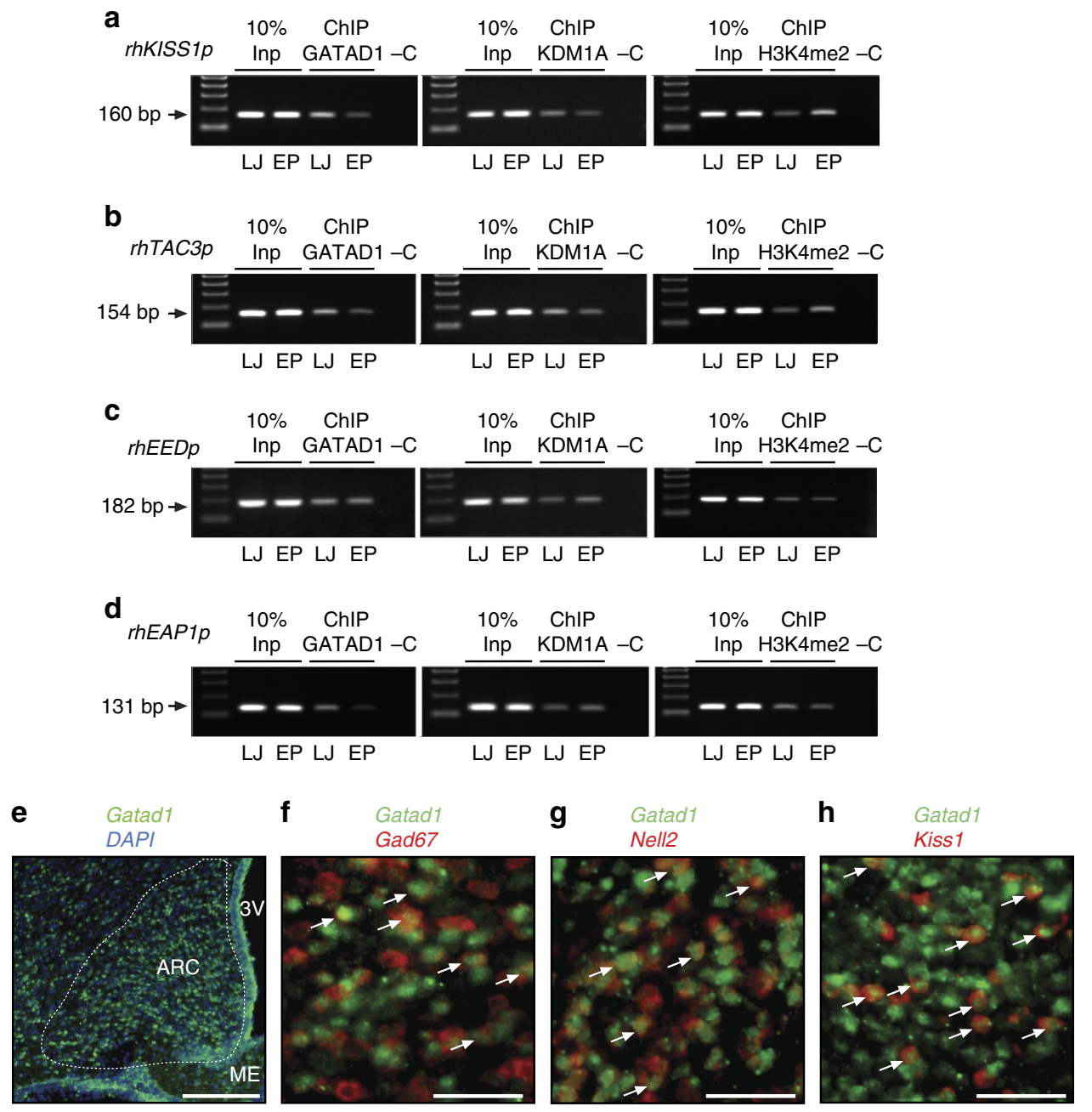

Figure 8 | GATAD1 and KDM1A association to the rhKISS1 and rhTAC3 promoters. (a) Decreased association of GATAD1 and KDM1A to, and increased $\mathrm{H} 3 \mathrm{~K} 4 \mathrm{me} 2$ abundance at the rhesus ( $\mathrm{rh}$ ) Kiss1 promoter in the MBH of female monkeys at the juvenile-pubertal transition. (b) Similar changes occur at the rhTAC3 promoter. (c) Absence of changes in GATAD1, KDM1A and H3K4me2 at the rhEED promoter. (d) Reduction of GATAD1 association to the rhEAP1 promoter in the absence of changes in KDM1A and H3K4me2 abundance. (e) Low-magnification image showing the presence of Gatad1 mRNA transcripts in the ARC of a 28-day-old late juvenile female rat as assessed by fluorescent in situ hybridization (FISH) using a Gatad1 cRNA labelled with FITC. Bar, $200 \mu \mathrm{m}$. (f) Detection of Gatad1 mRNA transcripts in a subpopulation of GABAergic neurons (examples denoted by arrows) identified by their content of GAD67 mRNA (detected using a digoxigenin-labelled Gad67 cRNA probe). (g) Gatad1 mRNA is also detected in a subpopulation of glutamatergic neurons (arrows) identified by the presence of Nell2 mRNA (detected with a digoxigenin-labelled Nell2 cRNA probe). (h) Most ARC kisspeptin neurons identified by the presence of Kiss1 mRNA contain Gatad1 mRNA transcripts (arrows). Bars in f-h, $50 \mu \mathrm{m}$.

Our results also show that primate-specific ZNFs do not contribute to regulating the timing of puberty in primates. Instead, puberty appears to be regulated by ZNFs, such as GATAD1 and ZNF573, less affected by lineage-specific diversification and expressed across mammalian species, including primates. The term 'primate-specific' refers to a group of $K R A B-Z N F$ and $Z N F$ genes that, resulting from lineage-specific evolutionary expansion and diversification, is unique to primates $^{23,24}$. The two largest clusters of primate-specific genes reside in the centromeric regions of chromosome 19 (p12-13.1) and chromosome 7 (7p11.2-q11.21). Although we did not detect changes in GATAD1 expression in the rat hypothalamus at puberty, GATAD1 was effective in delaying puberty in this species suggesting that ZNFs may be able to substitute for other ZNFs in the regulation of major biological processes such as puberty. This idea is supported by the finding of several ZNFs displaying reduced abundance in the monkey hypothalamus at puberty, and is consistent with the concept that the large number of ZNFs present in the mammalian genome is important for the 'fine tuning of transcriptional regulation within regulatory networks 25 .

GATAD1 is a particularly intriguing ZNF not only because it has been implicated in determining adult height ${ }^{14,15}$, but also because of its contribution to the epigenetic regulation of gene expression ${ }^{16}$. GATAD1 is considered to be a chromatin 'reader' because it associates to promoter regions containing $\mathrm{H} 3 \mathrm{~K} 4 \mathrm{me} 3$ to form a repressive complex ${ }^{16}$. H3K4me3 is a bona fide histone modification associated with active gene transcription ${ }^{26}$. For the foregoing reasons, we conducted a more detailed analysis of the potential mechanisms by which GATAD1 might induce transcriptional repression in the ARC using a reverse translation approach with the intact female rat serving as our in vivo model. This approach revealed that GATAD1 overexpression disrupts the normal changes in gene network connectivity that occur in the ARC as puberty approaches, preventing a juvenile-to-peripuberal organization geared for transcriptional activation and maintaining one less 'mature' that favours transcriptional silencing. GATAD1-imposed 
transcriptional silencing appears to be exerted at two levels. The first involves direct transcriptional repression of Kiss1 and Tac3, two genes expressed in the ARC and considered to be central to the pubertal increase in GnRH pulsatility. The second level of transcriptional silencing leads to a switch in the chromatin landscape from a configuration-favouring gene activation to another that promotes gene silencing. The presence of GATAD1 transcripts in kisspeptin neurons of the rat ARC provides morphological support to the concept that GATAD1 is a physiological regulator of the pubertal process.

Expression of both KISS1 and TAC3 increases in the monkey hypothalamus, as GATAD1 expression decreases during the JUV-PUB transition. That these events may be causally related is suggested by the results of our promoter assays showing that GATAD1 inhibits KISS1 and TAC3 promoter activity. This repression occurs in the absence of exogenously added co-repressors. Surprisingly, GATAD1 did not affect the promoter activity of other genes tested. This is perhaps due to the need of co-repressors or to a potential effect of GATAD1 on the body of these genes to recruit SETDB1, a methyltransferase that catalyses the synthesis of the repressive histone mark H3K9me3 (ref. 27).

Our gene network analysis predicted the contribution of several epigenetic domains to the regulation of puberty and the repression of this process by GATAD1. We pursued studies on one of these domains, because of its direct relevance to the epigenetic regulation of gene expression. According to our computational analysis, Kdm1a loses connectivity in the ARC during normal prepubertal development of the female rat and GATAD1 overexpression prevents this change. KDM1A (also known as LSD1), the lysine demethylase encoded by Kdm1a, specifically demethylates $\mathrm{H} 3 \mathrm{~K} 4 \mathrm{me} 2$ at gene promoters $^{19}$, and, therefore, is linked to gene repression ${ }^{19,20}$. Our in vitro finding that KDM1A recruitment is increased by GATAD1 overexpression while association of H3K4me2 to the KISS1 and TAC3 promoters is dramatically reduced, is consistent with the interpretation that GATAD1 reduces KISS1 and TAC3 gene activity in part by facilitating loss of $\mathrm{H} 3 \mathrm{~K} 4 \mathrm{me} 2$ from these promoters via gain of KDM1A. The decrease in GATAD1 and KDM1A association to the KISS1 and TAC3 promoters and the concomitant increase in $\mathrm{H} 2 \mathrm{~K} 4 \mathrm{me} 2$ abundance observed in the monkey $\mathrm{MBH}$ at the time of puberty, strongly support the in vitro results and suggest that these epigenetic changes are events normally associated with the time of primate puberty. Interestingly, GATAD1 and KDM1A appear to be interconnected in a broader context as sequence variation in genomic loci harbouring these genes has been shown to affect human height ${ }^{14,15}$.

Altogether, these results are consistent with the emerging concept that transcriptional repression of genes involved in the initiation of puberty resides at the heart of the molecular mechanism by which the hypothalamus holds the GnRH pulse generator in check and therefore prevents the premature activation of the pubertal process ${ }^{4}$. The polycomb (PcG) complex, known to play a major role in imposing gene silencing at various developmental stages ${ }^{28}$, was shown by one of our laboratories to prevent the premature initiation of puberty in the rat by repressing the transcriptional activity of Kiss1 in KNDy neurons ${ }^{4}$. The ability of GATAD1 to promote H3K4me2 demethylation at the KISS1 and TAC3 promoters suggests that ZNFs, instead of directly interacting with PcG proteins, may function to counteract the antagonistic effect of the Trithorax complex on PcG-mediated gene silencing. Mono, di and trimethylation of $\mathrm{H} 3$ at lysine 4 at target promoters are histone modifications associated with active transcription and catalysed by specific members of the Trithorax complex ${ }^{29,30}$.
Of the ZNFs we studied, only expression of GATAD1 exhibited an inverse developmental relationship with the centrally driven 'on-off-on' pattern of pulsatile GnRH release observed in highly evolved primates during the infant-juvenile and JUV-PUB transitions ${ }^{1}$. This finding implies that the ZNF components of the molecular switch that we posit, controls the pubertal reactivation of GnRH pulsatility, may not be identical to those comprising the switch that represses the GnRH pulse generator during the infant-juvenile transition. Unexpectedly, the decline in GnRH pulsatility during the INF-JUV transition was not accompanied by a decrease in either KISS1 or TAC3 mRNA levels. This is surprising in the view of an earlier study showing a greater number of ARC kisspeptin neurons in agonadal infantile male monkeys than in juveniles ${ }^{31}$. Also, hypogonadotropism was reported in an infant boy with a loss-of-function mutation of KISS1R/GPR54 (ref. 32). Clearly additional studies are required to unravel the regulation of $\mathrm{GnRH}$ secretion during primate infancy.

By identifying a cohort of ZNFs with decreasing hypothalamic expression during the JUV-PUB transition in rhesus monkeys, and mechanistically characterizing the mode of action of one of them in a rodent model, our results enable us to advance the notion that ZNFs are transcriptional regulators involved in the process by which epigenetic repression contributes to regulating the timing of primate puberty. We further propose that ZNFs may provide a layer of control linked to that provided by EAP1 and MKRN3, two ZNF and RING finger-containing proteins earlier shown to share puberty-regulatory functions in rodents and primates ${ }^{33,34}$. Whether a similar ZNF-mediated mechanism contributes to restraining puberty in rodents is not known, but the fact that rat puberty is delayed by human ZNFs suggests this to be the case.

\section{Methods}

Animals. Monkeys. For the study of the INF-JUV transition, 10 male rhesus monkeys bred by the Primate Core of the Center for Research in Reproductive Physiology, University of Pittsburgh were used. Ten juvenile male rhesus monkeys were also obtained from Three Springs Scientific (Perkasie, PA, USA) and maintained at the Center's Primate Core for the study of the JUV-PUB transition. The animals were housed under a controlled photoperiod (lights on, 0700-1900 h) in accordance with the National Institutes of Health (NIH) Guidelines for Care and Use of Laboratory Animals. The University of Pittsburgh Institutional Animal Care and Use Committee approved the experimental procedures. In addition, we used female rhesus monkeys (Macaca mulatta) obtained through the Oregon National Primate Research Center (ONPRC) Tissue Distribution Program. As in the case of male monkeys, these animals were classified into different stages of development This classification was based on the age of the animals and the pubertal stages reported by Watanabe and Terasawa ${ }^{35}$.

Rats. We used Sprague Dawley female rats at different phase of postnatal development, including postnatal day (PND)14, EJ period (PND21), LJ period (PND28), peripubertal period (PND29-35) and early adulthood (PND35-50). The use of rats was approved by the ONPRC Animal Care and Use Committee in accordance with the NIH guidelines for the use of animals in research. The animals were obtained from Charles River Laboratories international, Inc. (Wilmington, MA). They were housed in a room with controlled photoperiod (14/10 h light/dark cycle) and temperature $\left(23-25^{\circ} \mathrm{C}\right)$, and were allowed ad libitum access to tap water and pelleted rat chow.

Procedures involving monkeys. Surgical procedures. All animals for the study of the INF-JUV transition were bilaterally castrated between 2-6 days after birth using sterile technique under ketamine hydrochloride anaesthesia $\left(40 \mathrm{mg} \mathrm{kg}^{-1}\right.$ intramuscularly (i.m.); Ketaject, Phoenix Scientific, Inc., St Joseph, MO, USA) as reported previously ${ }^{36}$. In addition, local anaesthesia at the incision sites was achieved by the application of bupivacaine $\mathrm{HCl}$ (Marcaine, 0.5\%; Butler Schein Animal Health, Elizabethtown, PA, USA). Immediately before surgery, animals received an i.m. injection of penicillin (Pen-G, 40,000 $\mathrm{U} \mathrm{kg}^{-1}$; Phoenix Scientific, Inc.) Post-surgically, the infants received an i.m. injection of $2.5 \mathrm{mg}$ flunixin meglumine (Banamine, Butler Schein Animal Health, Elizabethtown, PA, USA) for analgesia. The infants were housed with their mothers in individual cages until they were killed between 1-3 (INF) and 6-11 (JUV) months of age to collect the hypothalamus (see below). 
All animals for the study of the JUV-PUB transition were bilaterally castrated as reported previously ${ }^{37}$ under isoflurane anaesthesia (1-2\% in oxygen; Abbott Animal House, North Chicago, IL, USA) using sterile technique between 16-18 months of age, that is, $18-20$ months before the initiation of puberty would be anticipated had the animals remained intact ${ }^{38}$. Animals were treated daily for 4 days starting on the morning before castration with an i.m. injection of Pen-G $\left(40,000 \mathrm{U} \mathrm{kg}^{-1}\right)$. Post-operative analgesia was achieved with twice daily i.m. injection of ketoprofen (Ketofen, $2 \mathrm{mg} \mathrm{kg}^{-1}$; Apothecon, Princeton, NJ, USA) for 4 days. Four animals were killed when they were around 19 months of age (JUV), four were killed when they were between 24-30 months of age (Early Pubertal, EP) and two were killed around 31 months of age (Late Pubertal, LP) to collect the hypothalamus. To collect the hypothalamus at the time of killing, animals from both studies were first sedated with an i.m. injection of ketamine $\left(20 \mathrm{mg} \mathrm{kg}^{-1}\right)$ followed by deep anaesthesia with intravenous sodium pentobarbital (Nembutal sodium solution, $\sim 25 \mathrm{mg} \mathrm{kg}^{-1}$; Abbott Laboratories, North Chicago, IL, USA) and placed in a stereotaxic head holder. Animals in the INF-JUV study were killed with a lethal intravenous dose of sodium pentobarbital $\left(40 \mathrm{mg} \mathrm{kg}^{-1}\right)$. Animals in the JUV-PUB study were killed with a mixture of pentobarbital and phenytoin (Beuthanasia-D, $1 \mathrm{ml} \mathrm{kg}^{-1}$; Butler Schein Animal Health, Elizabethtown, PA, USA).

Blood sampling. Weekly blood samples (1-2 ml) were collected via femoral venipuncture. For the first 6 months of the INF-JUV study, the mother was sedated with ketamine $\left(20 \mathrm{mg} \mathrm{kg}^{-1}\right.$, i.m) to remove the infant for sampling. Subsequently, the mother and infant were both sedated with ketamine to venipuncture the infant. For the JUV-PUB study, all animals were sedated with ketamine $\left(20 \mathrm{mg} \mathrm{kg}^{-1}\right)$ for venipuncture. Plasma was separated and stored at $-20^{\circ} \mathrm{C}$.

Tissue dissection. The brain was removed from the cranium and the hypothalamus isolated as a single block comprised of preoptic area (POA) and MBH. The hypothalamus was then bi-sectioned in the sagittal plane. One halfhypothalamus was further cut along the caudal boundary of the optic chiasm to deliver the POA and $\mathrm{MBH}$. The tissue was rapidly frozen by immersion in liquid nitrogen and stored at $-80^{\circ} \mathrm{C}$. A piece of cortical tissue (CTX, negative control) was also dissected, rapidly frozen and stored at $-80^{\circ} \mathrm{C}$.

\section{Procedures involving rats. Tissue collection. To measure changes in gene} expression that occur in the hypothalamus during juvenile development, the $\mathrm{MBH}$ of female rats was dissected by a rostral cut along the posterior border of the optic chiasm, a caudal cut immediately in front of the mammillary bodies, and two lateral cuts half-way between the medial eminence and the hypothalamic sulci. The thickness of the tissue fragment was about $2 \mathrm{~mm}$. On dissection, the tissues were immediately frozen on dry ice and stored at $-85^{\circ} \mathrm{C}$ until RNA extraction.

Stereotaxic delivery of lentiviral particles. To deliver LV particles carrying either a GATAD1 or a ZNF573 transgene to the ARC we used 21-day-old EJ female rats, using a procedure previously reported ${ }^{33,39}$. The animals were anaesthetized with a cocktail of ketamine $(25 \mathrm{mg})$, xylazine $(5 \mathrm{mg})$ and acepromazine $(1 \mathrm{mg}), 0.15 \mathrm{ml}$ per $100 \mathrm{~g}$ body weight (intraperitoneally), and positioned on a stereotaxic instrument (David Kopf Instruments, Tujunga, CA) with the incisor bar positioned at $+5 \mathrm{~mm}$. A total volume of $1 \mu \mathrm{l}$ of LV-GFP, LV-GATAD1 or LV-ZNF573 was injected bilaterally into the ARC at the rate of $250 \mathrm{nl} \mathrm{min}^{-1}$, using a $10 \mu \mathrm{l}$ Hamilton microsyringe connected to a Stoelting Stereotaxic microinjector (Stoelting, Wood Dale, IL). The coordinates used were: $0.3 \mathrm{~mm}$ lateral from midline, $0.2 \mathrm{~mm}$ anterior from bregma and $9.6 \mathrm{~mm}$ vertical from the surface of the brain, as reported ${ }^{33}$. The surgical procedure lasted about $15 \mathrm{~min}$. Following surgery, the animals were placed in a clean cage on a heating pad until returned to their cages. They were given an analgesic (Carprofen, $5 \mathrm{mg} \mathrm{kg}^{-1}$ ) and an antibiotic (Baytril $10 \mathrm{mg} \mathrm{kg}^{-1}$ ) for 3 days. At the time of killing, the location of the injections was determined by immunohistochemical detection of enhanced GFP (eGFP), as described below.

Evaluation of sexual maturation and oestrous cyclicity. To determine the changes in hypothalamic gene expression that occur during the INF-JUV period of sexual development, rats were euthanized at PND14, PND21 and PND28. According to criteria previously established ${ }^{40,41}$, PND21 animals are considered to be in the EJ phase of prepubertal development. At this time, the vagina is not yet patent and the uterine weight is $60 \mathrm{mg}$ or less, with no accumulation of intrauterine fluid. At PND28, the rats are in the LJ phase of prepubertal development; their vagina is closed and there are no signs of intrauterine fluid accumulation. Animals in this phase exhibit a diurnal change in pulsatile plasma LH levels, with the LH pulses becoming more pronounced in the afternoons ${ }^{42}$. Like in humans and monkeys, this is the first hormonal manifestation of the increase in central drive that initiates puberty ${ }^{43}$. To determine the effect of intrahypothalamic injections of ZNFs on the onset of puberty and subsequent oestrous cyclicity the animals were inspected daily for vaginal opening starting 5 days after the injections. Once vaginal opening occurred, vaginal lavages were performed daily to identify the occurrence of the first oestrus, which in rodents is manifested by a predominance of cornified cells. Although ovulation normally occurs on the day of oestrous, detection of cornified cells does not indicate that ovulation has occurred, unless vaginal cornification is followed by the appearance of a predominance of leukocytes. The presence of these cells defines the diestrous phase of the oestrous cycle, and indicates that a functional corpus luteum was formed after ovulation. For these reasons, the age at first ovulation was considered to have occurred only when the cornified cells were followed by at least 2 days of lavages containing mostly leukocytes.

RNA extraction. Total RNA was extracted from the MBH, POA and CTX of male and female rhesus monkeys using the RNeasy maxi kit (Qiagen, Valencia, CA) or from rat $\mathrm{MBH}$ using the RNeasy mini kit (Qiagen) following the manufacturer's instructions. To remove DNA contamination, RNA samples were treated by on-column DNAse digestion with the Qiagen RNase-free DNase set according to the manufacturer's protocol. RNA concentrations were determined by spectrophotometric trace (Nanodrop, ThermoScientific, Wilmington, DE).

Affymetrix arrays. Total RNA from rhesus monkeys was concentrated with the RNA Clean-up Kit-25 (Zymo Research Corporation, Irvine, CA) following the manufacturer's instruction to reach a minimum concentration of $500 \mathrm{ng} \mu \mathrm{l}^{-1}$. RNA integrity was assessed on an Agilent 2100 Bioanalyzer (Agilent Technologies, Inc., Santa Clara, CA) and found acceptable and of equal quality. Samples were amplified, labelled and hybridized to GeneChip Rhesus Macaque Genome Arrays (Affymetrix, Santa Clara, CA). Microarray assays were performed by the Affymetrix Microarray Core of the OHSU Gene Microarray Shared Resource.

Open array real-time PCR. $1,000 \mathrm{ng}$ of total RNA from rat MBH was reverse transcribed (RT) using the Omni RT Kit (Qiagen, Valencia, CA) in the presence of random hexamer primers (Invitrogen, Carlsbad, CA), as recommended by the manufacturer. The resulting complementary DNA ( $\mathrm{cDNA}$ ) was diluted four times with $\mathrm{H}_{2} \mathrm{O}$ and mixed with $2 \times$ TaqMan OpenArray Real-Time PCR Master Mix (Life Technologies, Grand Island, NY) at a ratio of 3.8:1.2 (PCR mix:cDNA). The mix was loaded into custom-made $(12 \times 224$ probes) OpenArray plates (for target genes see Supplementary Data 5; for probe numbers and lengths of amplicon see Supplementary Data 9) using the QuantStudio OpenArray AccuFill platform and the PCR reactions were performed in a QuantStudio $12 \mathrm{~K}$ Flex Real-Time PCR System (Applied Biosystems, Foster City, CA). Experiments were performed two times.

Real-time PCR. $400 \mathrm{ng}$ of total RNA were RT as described above. All real-time PCR reactions were performed using a Quanttudio $12 \mathrm{~K}$ Real-Time PCR system: threshold cycles (CTs) were detected by QuantStudio $12 \mathrm{~K}$ Flex software. Relative standard curves were constructed from serial dilutions of one reference sample cDNA (RT of 500 ng total RNA from one MBH sample serially diluted from $1 / 2$ to $1 / 500)$. The CTs from each sample was referred to the relative curve to estimate the mRNA content/sample and the values obtained were normalized for procedural losses using GAPDH mRNA (for monkey tissues) or peptidylprolyl isomerase A mRNA (for rat tissues) as the normalizing unit. To determine the relative abundance of the mRNAs of interest, we used the SYBR GreenER qPCR SuperMix system (Invitrogen, Carlsbad, CA). Primers for amplification (Supplementary Data 9) were designed using the PrimerSelect tool of DNASTAR 11 software (Madison, WI) or the NCBI online Primer-Blast program. PCR reactions were performed in a total volume of $10 \mu \mathrm{l}$, each reaction containing $1 \mu \mathrm{l}$ of diluted cDNA or a reference cDNA sample, $5 \mu \mathrm{l}$ of SYBR GreenER qPCR SuperMix and $4 \mu \mathrm{l}$ of primers mix $(300 \mathrm{nM}$ of each gene specific primer. The PCR conditions used were $95^{\circ} \mathrm{C}$ for $5 \mathrm{~min}$, followed by 40 cycles of $15 \mathrm{~s}$ at $95^{\circ} \mathrm{C}$ and $60 \mathrm{~s}$ at $60^{\circ} \mathrm{C}$. To confirm the formation of a single SYBR Green-labelled PCR amplicon, the PCR reaction was followed by a three-step melting curve analysis consisting of $15 \mathrm{~s}$ at $95^{\circ} \mathrm{C}$, $15 \mathrm{~s} 1 \mathrm{~min}$ at $60^{\circ} \mathrm{C}$, ramping up to $95^{\circ} \mathrm{C}$ at $0.5^{\circ} \mathrm{Cs} \mathrm{s}^{-1}$, detection every $0.5 \mathrm{~s}$ and finishing $15 \mathrm{~s}$ at $95^{\circ} \mathrm{C}$, as recommended by the manufacturer. Experiments were performed three times.

Affymetrix array analysis. Initial microarray analysis and quality control were performed by the Affymetrix Microarray Core using GCOS version 1.4.0.036 software (Affymetrix). After this initial analysis, the array CEL files provided were uploaded to the GeneSifter analysis platform (http://www.geospiza.com/Products/ AnalysisEdition.shtml) for further differential expression and functional analyses. Expression levels were normalized using GeneSifter's implementation of the Guanine-Cytosine robust multiarray analysis algorithm ${ }^{44}$. After filtering for absent probe calls, gene expression levels were visualized using the GeneSifter interface and compared for differential expression across time points using a one-way analysis of variance (ANOVA) analysis. In lieu of Affymetrix standard probe annotation, we used the annotations provided by Robert Nordgren (http:// www.unmc.edu/rhesusgenechip/).

Heat maps and expression density estimation plots. Gene expression data from microarray experiments were analysed in the R statistical environment (R Development Core Team) version 2.15 (http://www.R-project.org/) after data cleaning and normalization with GeneSifter as described above. Gene expressions were transformed to relative expression levels using the mean expression level of late juvenile samples as the reference. Heat maps of gene expression were generated using the heat map function of the gplots package and the RColorBrewer package, while density estimation of changes in the relative level of expression between 
developmental stages were plotted using R's density function with default parameters.

Open array analysis. We used the OpenArray qPCR platform to measure changes in relative expression for 224 genes studied during infantile-juvenile development (PND 14, 21 and 28) and after perturbing the system by overexpression of GATAD1 in the ARC (rats injected on PND21 and euthanized on PND28) (Supplementary Data 5 and 6). Raw data were extracted from the QuantStudio $12 \mathrm{~K}$ Flex software and analysed using R. CT values were converted to relative expression levels for further analysis using a standard delta-delta transformation. Probes that resulted in $50 \%$ or more of undetermined (UD) or flagged replicates were excluded from the study. Data were then used for gene co-expression and network analysis (see below).

Gene co-expression network analysis. After initial filtering of the OpenArray expression data as described above, we filtered remaining outliers and missing values from the data. The expression of these removed values was inferred with $\mathrm{k}$-nearest neighbour imputation using the impute $\mathrm{R}$ package. Due to the two runs of the OpenArray instrument necessary for data collection, genes that were excluded in one run were excluded from the other run, resulting in 147 genes viable for analysis across the three time points and GATAD1 overexpression experiments (Supplementary Data 6). The remaining expression values were then corrected for batch effects using the ComBat method of Johnson et al. ${ }^{45}$ as implemented in the sva $\mathrm{R}$ package. Thereafter, we constructed gene co-expression networks for each individual time point and the GATAD1 overexpression data set using a compressive-sensing-based network ensemble methodology developed by our group. We generated all possible one-gene Pearson partial correlation matrices (for example, for $n$ genes, $n$ matrices are produced, with the $n$th matrix representing the pairwise partial correlations of expression for all genes other than $n$ when accounting for the correlation of $n$ with both genes in a pair) from the data using the $\mathrm{R}$ ppcor package. We then utilized the CLIME algorithm ${ }^{46}$ as implemented in the $\mathrm{R}$ package clime to approximate inverse matrices for each partial correlation matrix in the ensemble. Twenty inverse matrices were estimated for each partial correlation matrix for twenty equi-spaced values of the CLIME regularization parameter lambda between 1 and 0.01 . We then selected the inverse matrix from the set that resulted in a network with a node degree distribution best approximating a scale-free distribution, filtered the edges in the resultant network based on a cutoff threshold of the 10th percentile of absolute edge weight in the inverse matrix. The lambda range and cutoff value were determined using empirical analysis of in silico simulated expression data (unpublished) to minimize false positives and negatives. Co-expression relationships that occur in $95 \%$ or more of the resultant networks were then included in the overall network (Supplementary Data 10), under the premise that edges appearing in this fraction of the total occurrences of all edges in the derived network are more likely to represent relatively direct or extremely strong co-expression relationships between the genes rather than spurious correlations or coregulation by other factors. Networks were visualized, analysed and compared using $\mathrm{R}$ and Cytoscape 3.1.1 (www.cytoscape.org).

Functional annotation of gene sets. In addition to analysing the structure of individual networks, the top 20 genes (including ties for the 20th position) losing or gaining network connectivity during prepubertal development were selected for Gene Ontology enrichment analysis using the DAVID tool ${ }^{47,48}$; gain and loss were defined as the sum of changes in node degree for a gene between each pair of the networks derived from the three time points assessed. In addition, we repeated this procedure for the top 20 genes losing or gaining connectivity in the network inferred from the GATAD1 overexpression data set as compared with the PND28 network. Overrepresented annotation categories for each set of genes were defined as categories with a $P$ value of 0.05 or less as reported by DAVID's modified Fisher Exact Test procedure after Benjamini-Hochberg correction for multiple testing was applied. Genes were mapped to human orthologs due to the superior functional annotation of the human genome, and compared against the human annotation as a background.

Preparation of lentiviruses for ZNF overexpression. To generate lentiviral particles expressing human GATAD1 or human ZNF573 the coding regions of these genes were purchased from OriGene Technologies (Rockville, MD). Both were provided as plasmids, GATAD1 inserted into the EcoRI-XhoI sites of pCMV6-AC, and ZNF573 into the Sgfl-MluI sites of pCMV-Entry (RC209422). GATAD1 was cloned into the BamHI-SmaI sites of the lentiviral vector LV-Ief via In-Fusion PCR cloning (http://www.clontech.com/US/Products/Cloning_and_ Competent_Cells/Cloning_Kits/Cloning_Kits-HD-Liquid) using the primers described in Supplementary Data 9. These primers encompass the juncture between the GATAD1 cDNA and LV-Ief. The LV-IEF plasmid derives from the plasmid pLVI IRES ${ }^{49,50}$ in which the IRES element connecting the gene of interest to eGFP was replaced with the promoter of human elongation factor to drive eGFP expression independent of the inserted gene. pLVI-IRES is a third generation plasmid, in which the promoter sequences of the $5^{\prime}$-LTR were replaced by the cytomegalovirus (CMV) promoter resulting in the formation of a heterologous
U3 promoter ${ }^{51,52}$. ZNF573 was excised from pCMV-Entry by first linearizing the plasmid with FseI and then blunting the FseI site with T4 DNA polymerase followed by BamHI digestion. The resulting fragment was then cloned into the BamHI-SmaI sites of LV-Ief. The coding region of hGATAD1 is tagged with the human influenza HA and ZNF573 is tagged with both MYC and a FLAG epitope for easy detection by western immunoblotting. To enhance transgene expression ${ }^{53}$ we inserted the rat insulin II intron A sequence ${ }^{54}$ between the CMV promoter and the GATAD1/ZNF573 cDNAs. This heterologous intron has been previously used in transgenic mice ${ }^{53,55}$.

Infective lentiviral particles were produced as previously described ${ }^{33,49}$. In brief, we used the calcium phosphate method to cotransfect $293 \mathrm{~T}$ cells with four plasmids; the LV vector, pLP1, pLP2 and pLPv (Invitrogen). Plasmid pLP1 expresses gag and pol; pLP2 expresses Rev; and pLPv expresses the VSVG envelope protein. The viral concentration was increased by ultracentrifugation, and the titre of the viral stock was determined by infection of naive 293T cells. Following infection with serial dilutions of the viral stock, the percentage of GFP-positive (fluorescent) cells was determined by flow cytometry. The titre was expressed as transducing units per millilitre $\left(\mathrm{TU} \mathrm{ml}^{-1}\right)$.

Functional promoter assays. To determine whether ZNF proteins alter the transcriptional activity of putative target genes (KISS1, TAC3, Ttf1, Eed, EAP1, PEnk and Viaat) we transfected Neuro2A cells (N2A, ATCC, Manassas, VA) with luciferase reporter constructs ${ }^{56}$ containing the $5^{\prime}$ flanking region of these genes, in addition to the LV-GATAD1 or LV-ZNF573 constructs mentioned above. The cells (ATCC, Manassas, VA) were cultured in a humidified atmosphere containing 5\% $\mathrm{CO}_{2}$ and $37^{\circ} \mathrm{C}$. They were maintained in DMEM containing high glucose ( $4.5 \mathrm{gl}^{-1}$; Sigma), supplemented with $10 \%$ fetal bovine serum (Invitrogen), Glutamine (Sigma), $100 \mathrm{U} \mathrm{ml}^{-1}$ penicillin, and $100 \mathrm{\mu g} \mathrm{ml}^{-1}$ streptomycin (Invitrogen). For the assays, the cells (400,000 cells per well) were seeded onto 24-well plates in DMEM containing $10 \%$ fetal bovine serum. Twenty-four hours later, the reporter constructs (all of them in the luciferase reporter plasmid pGL2) were transiently co-transfected along with LV-GATAD1 or LV-ZNF573 for $5 \mathrm{~h}$ using Lipofectamine 2000 (Invitrogen) at a ratio ( $1 \mu \mathrm{g}$ DNA:2.5 $\mu$ l Lipofectamine 2000) in Optimem (Invitrogen). After transfection, the cells were returned to serum containing DMEM medium; $24 \mathrm{~h}$ later, they were harvested and assayed for luciferase activity using the Firefly Luciferase Glow Assay Kit (Pierce, Rockford, IL). The assay was performed in opaque 96-well plates and light emission measured in a Spectramax M5 microplate reader (Molecular Devices, Sunnyvale, CA). Transfection efficiency was normalized by co-transfecting the plasmid CMV-Sport- $\beta$-gal (Invitrogen) at $10 \mathrm{ng} \mathrm{ml}^{-1}$ and determining $\beta$-Galactosidase activity using the Tropix Galacto Light Plus (ABI) as reported earlier ${ }^{33}$. Experiments were performed three times.

Western blots. To verify the ability of the LV-GATAD1 and LV-ZNF573 constructs to produce a mature protein, we transfected $293 \mathrm{~T}$ cells with the constructs using Lipofectamine 2000 (Invitrogen) and lysed the cells $48 \mathrm{~h}$ later with $500 \mu \mathrm{l}$ of freshly prepared RIPA buffer $(25 \mathrm{mM}$ Tris, $\mathrm{pH} 7.4,1 \%$ Triton X-100, $1 \%$ sodium deoxycholate, $0.1 \%$ SDS, $150 \mathrm{mM} \mathrm{NaCl}, 50 \mathrm{mM} \beta$-glycerophosphate, $1 \mathrm{mM}$ sodium pyrophosphate, $1 \mathrm{mM}$ sodium orthovanadate, $10 \mu \mathrm{g} \mathrm{ml}^{-1}$ leupeptin and Pepstatin A, $10 \mu \mathrm{g} \mathrm{ml}^{-1}$ aprotinin and $100 \mu \mathrm{g} \mathrm{ml}^{-1}$ PMSF). Protein concentrations were estimated using the Pierce $660 \mathrm{~nm}$ Protein Assay (Pierce, Rockford, IL). Samples were boiled for $5 \mathrm{~min}$ in sample buffer and then fractionated in an $8-16 \%$ precast SDS-PAGE gel (Invitrogen, Carlsbad, CA). After electrophoresis at $130 \mathrm{~V}$ for $2 \mathrm{~h}$, the proteins were transferred for $4 \mathrm{~h}$ at $4{ }^{\circ} \mathrm{C}$ onto a polyvinylidene difluoride membrane (Millipore, Billerica, MA). The membrane was blocked in $5 \%$ non-fat milk for $1 \mathrm{~h}$, before adding the appropriate antibodies (Supplementary Table 1). After an overnight incubation at $4{ }^{\circ} \mathrm{C}$ with the primary antibody, the membranes were incubated with a species-specific anti IgG-horseradish peroxidase antibody ( $1 \mathrm{~h}$ at room temperature, 1:10,000; Invitrogen). The signal was developed by enhanced chemiluminescence using the western lightning chemiluminescence substrate (Pierce).

ChIP assay. To assess the recruitment of GATAD1 to target-specific gene promoters, as well as the association of histone marks to these regions, we performed ChIP assays using chromatin extracted from either 293T cells transfected with the HA-tagged LV-ODAG construct, or hypothalamic tissue $(\mathrm{MBH})$ derived from two female rhesus monkeys. One of these animals ( 2 years and 99 days age) was in the late juvenile phase of postnatal development; the other ( 3 years and 73 days of age) was in the early puberty phase. The ChIP procedure was described previously by us $4,56,57$, and was carried out with minimal modifications. Cells were harvested for ChIP $48 \mathrm{~h}$ after transfection. The cells were washed once in ice-cold PBS containing a protease inhibitor cocktail (1 mM phenylmethylsulfonylfluoride, $7 \mu \mathrm{g} \mathrm{ml}^{-1}$ aprotinin, $0.7 \mu \mathrm{g} \mathrm{ml}^{-1}$ pepstatin A, $0.5 \mu \mathrm{g} \mathrm{ml}^{-1}$ leupeptin), a phosphatase inhibitor cocktail (1 mM $\beta$-glycerophosphate, $1 \mathrm{mM}$ sodium pyrophosphate and $1 \mathrm{mM}$ sodium fluoride), an HDAC inhibitor ( $20 \mathrm{mM}$ sodium butyrate). Cross-linking was performed by incubating the cell suspension or $\mathrm{MBH}$ fragments in $1 \%$ formaldehyde for $10 \mathrm{~min}$ at room temperature. After two additional washing steps in PBS, the cells and MBH fragments were lysed with $200 \mu \mathrm{l}$ SDS buffer $(0.5 \%$ SDS, $50 \mathrm{mM}$ Tris- $\mathrm{HCl}, 10 \mathrm{mM}$ 
EDTA) containing protease, phosphatase and HDAC inhibitors and sonicated for $45 \mathrm{~s}$ to yield chromatin fragments of $\sim 500 \mathrm{bp}$ using a Fisher Scientific FB 705 sonicator. Size fragmentation was confirmed by agarose gel electrophoresis. The sonicated chromatin was clarified by centrifugation at $16,200 \mathrm{~g}$ for $10 \mathrm{~min}$ at $4{ }^{\circ} \mathrm{C}$, brought up to $1 \mathrm{ml}$ in Chip Dilution Buffer (16.7 mM Tris-HCl, $\mathrm{pH} 8.1,150 \mathrm{mM}$ $\mathrm{NaCl}, 1.2 \mathrm{mM}$ EDTA, $1.1 \%$ Triton X-100 and $0.01 \%$ SDS) containing protease, phosphatase and HDAC inhibitors and stored at $-80^{\circ} \mathrm{C}$ for subsequent IP For this step, chromatin was pre-cleared with Protein A/G beads (Dynabeads, Invitrogen, Carlsbad, CA) for $1 \mathrm{~h}$ at $4{ }^{\circ} \mathrm{C} .100 \mu \mathrm{l}$ aliquots of chromatin were then incubated with $5 \mu \mathrm{g}$ antibodies. The antibodies used are described in Supplementary Table 1. Antibody-chromatin complexes and $25 \mu \mathrm{l}$ of protein A or $\mathrm{G}$ beads solution (Dynabeads) were incubated at $4{ }^{\circ} \mathrm{C}$ overnight with gentle agitation. Immunocomplexes were washed sequentially with $0.5 \mathrm{ml}$ low-salt wash buffer (20 mM Tris-HCl, pH 8.1, $150 \mathrm{mM} \mathrm{NaCl}, 2$ mM EDTA, 1\% Triton X-100 and $0.1 \%$ SDS), high-salt wash buffer $(20 \mathrm{mM}$ Tris- $\mathrm{HCl}, \mathrm{pH} 8.1,500 \mathrm{mM} \mathrm{NaCl}$, $2 \mathrm{mM}$ EDTA, $1 \%$ Triton X-100 and $0.1 \%$ SDS), LiCl buffer (10 mM Tris-HCl, $\mathrm{pH}$ 8.1, $250 \mathrm{M} \mathrm{LiCl}, 1 \%$ Nonidet P-40, $1 \%$ sodium deoxycholate and $1 \mathrm{mM}$ EDTA) and with TE buffer $(10 \mathrm{mM}$ Tris- $\mathrm{HCl}, \mathrm{pH} 8.0$ and $1 \mathrm{mM}$ EDTA). The immunocomplexes were eluted with $100 \mu \mathrm{l}$ of $0.1 \mathrm{M} \mathrm{NaHCO} 3$ and $1 \%$ SDS at $65^{\circ} \mathrm{C}$ for $45 \mathrm{~min}$. Cross-linking was reversed by adding $4 \mu \mathrm{l}$ of $5 \mathrm{M} \mathrm{NaCl}$ and incubating at $95^{\circ} \mathrm{C}$ for $30 \mathrm{~min}$. DNA was recovered by using the ChIP DNA Clean and Concentrator columns (Zymo Research, Irvine, CA) and stored at $-80^{\circ} \mathrm{C}$ until subsequent PCR analysis. All chemicals were purchased from Sigma-Aldrich (St Louis, MO, USA).

\section{PCR detection of ChIP DNA. Genomic regions of interest were amplified by} qPCR. Accession numbers of the genes analysed as well as the chromosomal position of the $5^{\prime}$-flanking region amplified, using the position of the transcription start site as the reference point, are shown in Supplementary Data 9. The primer sequences (Eurofins MWG Operon, Huntsville, AL) used to detect the DNA fragment of interest in the immunoprecipitated DNA are also shown in Supplementary Data 9. For semi quantitative detection, PCR reactions were performed using $1 \mu \mathrm{l}$ of each IP and Input samples and SybrGreen ER (Invitrogen, Carlsbad, CA) in a volume of $10 \mu$. The thermocycling conditions used were: $95^{\circ} \mathrm{C}$ for $5 \mathrm{~min}$, followed by 40 cycles of $15 \mathrm{~s}$ at $95^{\circ} \mathrm{C}$ and $60 \mathrm{~s}$ at $60^{\circ} \mathrm{C}$. Data are expressed as \% of IP signal / Input signal. Experiments were performed three times. For qualitative detection, PCR reactions were performed using $1 \mu \mathrm{l}$ of each IP and Input samples and HotStart Taq polymerase (Qiagen, Valencia, CA) in a volume of $25 \mu \mathrm{l}$. The thermocycling conditions used were: $95^{\circ} \mathrm{C}$ for $5 \mathrm{~min}$, followed by 35 cycles of $30 \mathrm{~s}$ at $95^{\circ} \mathrm{C}, 60 \mathrm{~s}$ at $60^{\circ} \mathrm{C}$ and $60 \mathrm{~s}$ at $72^{\circ} \mathrm{C} .12 \mu \mathrm{l}$ of PCR product were run in a $1.6 \%$ Agarose-TBE gel (uncropped images are supplied as Supplementary Figs 9 and 10).

Immunohistofluorescence. To verify the location of microinjections targeting GFP-expressing lentiviruses to the ARC of the hypothalamus, we perfusion-fixed the brain of the injected rats $22-25$ days after the initial infection with $4 \%$ paraformaldehyde-PBS pH 7.4, blocked the portion of the brain containing the hypothalamus, and serially sectioned the blocks at $30 \mu \mathrm{m}$ intervals. The sections were then incubated overnight at $4{ }^{\circ} \mathrm{C}$ with goat polyclonal antibodies against GFP (Abcam, Cambridge, MA; 1:2,000 dilution), and the reaction was developed the next day to a green colour using Alexa 488 donkey antigoat IgG (Invitrogen, 1:500). Fluorescent images were acquired with an AxioImager A2 Zeiss fluorescent microscope.

Fluorescent in situ hybridization. The brains from two late juvenile 28-day-old female rats were fixed by intracardiac perfusion of $4 \%$ paraformaldehyde borate buffer, $\mathrm{pH} 9.5$, and were processed for hybridization histochemistry, as previously described ${ }^{58-61}$. We used the double fluorescence in situ hybridization procedure described by Watakabe et al. ${ }^{62}$ employing four different cRNA probes. Three of them ( $r$ Kiss1, $r$ Gad67 and $r$ Nell 2) were labelled with digoxigenin-11-UTP (Dig). The fourth probe (rGatad1) was labelled with fluorescein-12-UTP (FITC). The Kiss 1 cRNA probe was transcribed from a $379 \mathrm{bp}$ rat Kiss $1 \mathrm{cDNA}$ corresponding to nucleotides (nt) 1-379 in rat Kiss1 mRNA ${ }^{63}$, the GAD67 cRNA was generated by in vitro transcription of a $220 \mathrm{bp}$ cDNA template complementary to nt 303-523 in the coding region of rat Gad67 mRNA ${ }^{64}$, and the Nell 2 cRNA probe was transcribed from a $333 \mathrm{bp}$ cDNA corresponding to nt 548-880 in rat Nell2 $\mathrm{mRNA}^{21}$. Finally, the rGatad1 cRNA probe was transcribed from a cDNA template corresponding to nt 223-570 in the coding region of rGatad1 mRNA. The labelling reactions were performed in a $10 \mu \mathrm{l}$ volume, as reported ${ }^{65}$. Control sections were incubated with sense probes transcribed from the same plasmid, but linearized on the $3^{\prime}$-end to transcribe the coding strand of the cDNA template. The fluorescent in situ hybridization procedure used was identical to that previously described by our laboratory ${ }^{4}$.

Gonadotropin assays. Plasma levels of LH and FSH were determined using homologous RIAs described previously ${ }^{66,67}$. For the LH assay, recombinant cynomolgus (rc) LH (AFP6936A) was used as the standard and radioiodinated trace and a polyclonal rabbit antiserum (AFP342994) raised against rcLH as the first antibody. For the FSH assay, rcFSH (AFP6940A) was used as the standard and radioiodinated trace and a polyclonal rabbit antiserum (AFP782594) raised against rcFSH as the first antibody. The mean sensitivity of the LH and FSH assay ranged between $0.15-1.5 \mathrm{ng} \mathrm{ml}^{-1}$ and $0.1-0.7 \mathrm{ng} \mathrm{ml}^{-1}$, respectively, depending on the volume of plasma used for the determination. Intra- and inter-assay coefficients of variation were less than 6.9 and $15.8 \%$ for $\mathrm{LH}$ and 9.8 and $15.3 \%$ for FSH, respectively. Gonadotropin concentrations below detection were assigned a value equivalent to the minimum detectable concentration.

Statistics. All statistical analyses were performed using SigmaStat software (Systat Software Inc., San Jose, CA). The differences between several groups were analysed by ANOVA followed by the Student-Newman-Keuls multiple comparison test for unequal replications. The Student's $t$-test was used to compare two groups. When comparing percentages, groups were subjected to arc-sine transformation before statistical analysis to convert them from a binomial to a normal distribution ${ }^{68}$. For an overall analysis of the real-time PCR data, the results were analysed using paired Student's $t$-tests (two groups). Statistical significance of the connectivity that exists between ZNF573 and GATAD1 to menarche-associated genes was assessed relative to the distribution of their connectivity in an ensemble of 1,000 networks based on randomly selected gene set as described ${ }^{69}$. Significance of the overlap detected between the top 20 genes gaining and losing connectivity over the PND14/21/28 co-expression networks and the top 20 genes gaining and losing connectivity between the GATAD1 overexpression and PND28 co-expression networks was assessed by using a hypergeometric test to determine the probability of selecting the same number of overlapping genes in 20 draws from 147 genes without replacement by chance. A $P$ value of $<0.05$ was considered statistically significant. The sample size was selected based on power analyses performed using the s.d. that we normally observe when measuring the parameters examined in this study and an $n=6$ per group. These analyses provide at least $80 \%$ (type II error $=0.124$ ) power to detect two effect sizes using either ANOVA or two-sided two-sample $t$-test with a significance level of 0.05 .

\section{References}

1. Plant, T. M., Terasawa, E. \& Witchel, S. F. in Physiology of Reproduction. eds. Plant, T. M. \& Zelesnick, A. J.) 1487-1536 (Elsevier, 2015).

2. Wakabayashi, Y. et al. Neurokinin B and dynorphin A in kisspeptin neurons of the arcuate nucleus participate in generation of periodic oscillation of neural activity driving pulsatile gonadotropin-releasing hormone secretion in the goat. J. Neurosci. 30, 3124-3132 (2010).

3. Cheng, G., Coolen, L. M., Padmanabhan, V., Goodman, R. L. \& Lehman, M. N. The kisspeptin/neurokinin $\mathrm{B} /$ dynorphin (KNDy) cell population of the arcuate nucleus: sex differences and effects of prenatal testosterone in sheep. Endocrinology 151, 301-311 (2010).

4. Lomniczi, A. et al. Epigenetic control of female puberty. Nat. Neurosci. 16, 281-289 (2013).

5. Messina, D. N., Glasscock, J., Gish, W. \& Lovett, M. An ORFeome-based analysis of human transcription factor genes and the construction of a microarray to interrogate their expression. Genome Res. 14, 2041-2047 (2004).

6. Urrutia, R. KRAB-containing zinc-finger repressor proteins. Genome Biol. 4, 231 (2003).

7. Krishna, S. S., Majumdar, I. \& Grishin, N. V. Structural classification of zinc fingers: survey and summary. Nucleic Acids Res. 31, 532-550 (2003).

8. Huntley, S. et al. A comprehensive catalog of human KRAB-associated zinc finger genes: insights into the evolutionary history of a large family of transcriptional repressors. Genome Res. 16, 669-677 (2006).

9. Perry, J. R. et al. Meta-analysis of genome-wide association data identifies two loci influencing age at menarche. Nat. Genet. 41, 648-650 (2009).

10. Elks, C. E. et al. Thirty new loci for age at menarche identified by a metaanalysis of genome-wide association studies. Nat. Genet. 42, 1077-1085 (2010).

11. Perry, J. R. et al. Parent-of-origin-specific allelic associations among 106 genomic loci for age at menarche. Nature 514, 92-96 (2014).

12. Tsuruga, T. et al. Ocular development-associated gene (ODAG), a novel gene highly expressed in ocular development. Gene 290, 125-130 (2002).

13. Theis, J. L. et al. Homozygosity mapping and exome sequencing reveal GATAD1 mutation in autosomal recessive dilated cardiomyopathy. Circ. Cardiovasc. Genet. 4, 585-594 (2011).

14. Gudbjartsson, D. F. et al. Many sequence variants affecting diversity of adult human height. Nat. Genet. 40, 609-615 (2008).

15. Lango, A. H. et al. Hundreds of variants clustered in genomic loci and biological pathways affect human height. Nature 467, 832-838 (2010).

16. Vermeulen, M. et al. Quantitative interaction proteomics and genome-wide profiling of epigenetic histone marks and their readers. Cell 142, 967-980 (2010).

17. Chang, Y. H. \& Tomlin, C. Data driven graph reconstruction using compressive sensing. 51st IEEE Conference on Decision and Control (2012) Ref Type: Abstract.

18. Krishnan, S., Horowitz, S. \& Trievel, R. C. Structure and function of histone H3 lysine 9 methyltransferases and demethylases. Chem. Bio. Chem. 12, 254-263 (2011).

19. Shi, Y. et al. Histone demethylation mediated by the nuclear amine oxidase homolog LSD1. Cell 119, 941-953 (2004). 
20. Bestor, T. H. Gene silencing. Methylation meets acetylation. Nature 393, 311-312 (1998).

21. Ha, C. M. et al. NELL2, a neuron-specific EGF-like protein, is selectively expressed in glutamatergic neurons and contributes to the glutamatergic control of GnRH neurons at puberty. Neuroendocrinology 88, 199-211 (2008).

22. Mostafavi, S., Ray, D., Warde-Farley, D., Grouios, C. \& Morris, Q. GeneMANIA: a real-time multiple association network integration algorithm for predicting gene function. Genome Biol. 9(Suppl 1): S4 (2008).

23. Hamilton, A. T. et al. Evolutionary expansion and divergence in the ZNF91 subfamily of primate-specific zinc finger genes. Genome Res. 16, 584-594 (2006).

24. Nowick, K., Hamilton, A. T., Zhang, H. \& Stubbs, L. Rapid sequence and expression divergence suggest selection for novel function in primate-specific KRAB-ZNF genes. Mol. Biol. Evol. 27, 2606-2617 (2010).

25. Hamilton, A. T., Huntley, S., Kim, J., Branscomb, E. \& Stubbs, L. Lineage-specific expansion of KRAB zinc-finger transcription factor genes: implications for the evolution of vertebrate regulatory networks. Cold Spring Harb. Symp. Quant. Biol. 68, 131-140 (2003).

26. Berger, S. L. The complex language of chromatin regulation during transcription. Nature 447, 407-412 (2007).

27. Frietze, S., O'Geen, H., Blahnik, K. R., Jin, V. X. \& Farnham, P. J. ZNF274 recruits the histone methyltransferase SETDB1 to the 3' ends of ZNF genes. PLOS ONE 5, e15082 (2010).

28. Schwartz, Y. B. \& Pirrotta, V. Polycomb silencing mechanisms and the management of genomic programmes. Nat. Rev. Genet. 8, 9-22 (2007).

29. Schuettengruber, B., Martinez, A. M., Iovino, N. \& Cavalli, G. Trithorax group proteins: switching genes on and keeping them active. Nat. Rev. Mol. Cell Biol. 12, 799-814 (2011).

30. Shilatifard, A. The COMPASS family of histone H3K4 methylases: mechanisms of regulation in development and disease pathogenesis. Annu. Rev. Biochem. 81, 65-95 (2012).

31. Ramaswamy, S., Dwarki, K., Ali, B., Gibbs, R. B. \& Plant, T. M. The decline in pulsatile GnRH release, as reflected by circulating LH concentrations, during the infant-juvenile transition in the agonadal male rhesus monkey (Macaca mulatta) is associated with a reduction in kisspeptin content of KNDy neurons of the arcuate nucleus in the hypothalamus. Endocrinology 154, 1845-1853 (2013).

32. Semple, R. K. et al. Two novel missense mutations in g protein-coupled receptor 54 in a patient with hypogonadotropic hypogonadism. J. Clin. Endocrinol. Metab. 90, 1849-1855 (2005).

33. Heger, S. et al. Enhanced at puberty 1 (EAP1) is a new transcriptional regulator of the female neuroendocrine reproductive axis. J. Clin. Invest. 117, 2145-2154 (2007).

34. Abreu, A. P. et al. Central precocious puberty caused by mutations in the imprinted gene MKRN3. N. Engl. J. Med. 368, 2467-2475 (2013).

35. Watanabe, G. \& Terasawa, E. In vivo release of luteinizing hormone releasing hormone increases with puberty in the female rhesus monkey. Endocrinology 125, 92-99 (1989).

36. El, M. M., Sahu, A. \& Plant, T. M. Changes in hypothalamic gene expression associated with the arrest of pulsatile gonadotropin-releasing hormone release during infancy in the agonadal male rhesus monkey (Macaca mulatta). Endocrinology 141, 3273-3277 (2000).

37. Ramaswamy, S. Pubertal augmentation in juvenile rhesus monkey testosterone production induced by invariant gonadotropin stimulation is inhibited by estrogen. J. Clin. Endocrinol. Metab. 90, 5866-5875 (2005).

38. Plant, T. M. A study of the role of the postnatal testes in determining the ontogeny of gonadotropin secretion in the male rhesus monkey (Macaca mulatta). Endocrinology 116, 1341-1350 (1985).

39. Rage, F. et al. Targeting transforming growth factor $\alpha$ expression to discrete loci of the neuroendocrine brain induces female sexual precocity. Proc. Natl Acad. Sci. USA 94, 2735-2740 (1997).

40. Ojeda, S. R. \& Urbanski, H. F. in The Physiology of Reproduction 2nd edn, Vol 2 (eds. Knobil, E. \& Neill, J. D.) 363-409 (Raven Press, 1994).

41. Ojeda, S. R. \& Skinner, M. K. in The Physiology of Reproduction 3rd edn (ed. Neill, J. D.) 2061-2126 (Academic Press/Elsevier, 2006).

42. Urbanski, H. F. \& Ojeda, S. R. The juvenile-peripubertal transition period in the female rat: establishment of a diurnal pattern of pulsatile luteinizing hormone secretion. Endocrinology 117, 644-649 (1985).

43. Ojeda, S. R. \& Terasawa, E. in Hormones, Brain and Behavior Vol 4 (eds. Pfaff, D. et al.) 589-659 (Elsevier, 2002).

44. Wu, Z., Irizarry, R. A., Glentleman, R., Martinex-Murillo, F. \& Spencer, F. A model based background adjustment for oligonucleotide expression array. J. Am. Stat. Assoc. 99, 909 (2004)

45. Johnson, W. E., Li, C. \& Rabinovic, A. Adjusting batch effects in microarray expression data using empirical Bayes methods. Biostatistics 8, 118-127 (2007).

46. Cai, T. T., Liu, W. \& Luo, X. A constraind L1 minimization approach for sparse precision matrix estimation. J. Am. Stat. Assoc. 106, 594-607 (2011).

47. Huang, d. W., Sherman, B. T. \& Lempicki, R. A. Bioinformatics enrichment tools: paths toward the comprehensive functional analysis of large gene lists. Nucleic Acids Res. 37, 1-13 (2009).
48. Huang, d. W., Sherman, B. T. \& Lempicki, R. A. Systematic and integrative analysis of large gene lists using DAVID bioinformatics resources. Nat. Protoc 4, 44-57 (2009).

49. Dissen, G. A. et al. In vivo manipulation of gene expression in non-human primates using lentiviral vectors as delivery vehicles. Methods. 49, 70-77 (2009).

50. Dissen, G. A. et al.in Controlled Genetic Manipulations. (ed. Morozov, A.) 69-96 (Springer Science + Business Media in their Neuromethods series formerly published by Humana Press, 2012).

51. Dull, T. et al. A third-generation lentivirus vector with a conditional packaging system. J. Virol. 72, 8463-8471 (1998).

52. Follenzi, A., Ailles, L. E., Bakovic, S., Geuna, M. \& Naldini, L. Gene transfer by lentiviral vectors is limited by nuclear translocation and rescued by HIV-1 pol sequences. Nat. Genet. 25, 217-222 (2000).

53. Palmiter, R. D., Sandgren, E. P., Avarbock, M. R., Allen, D. D. \& Brinster, R. L. Heterologous introns can enhance expression of transgenes in mice. Proc. Natl Acad. Sci. USA 88, 478-482 (1991).

54. Lomedico, P. et al. The structure and evolution of the two nonallelic rat preproinsulin genes. Cell 18, 545-558 (1979).

55. Hoyle, G. W. et al. Hyperinnervation of the airways in transgenic mice overexpressing nerve growth factor. Am. J. Respir. Cell Mol. Biol. 18, 149-157 (1998).

56. Mueller, J. K. et al. Transcriptional regulation of the human KiSS1 gene. Mol. Cell. Endocrinol. 342, 8-19 (2011).

57. Mueller, J. K. et al. Transcription of the human EAP1 gene is regulated by upstream components of a puberty-controlling Tumor Suppressor Gene network. Mol. Cell. Endocrinol. 351, 184-198 (2012).

58. Simmons, D. M., Arriza, J. L. \& Swanson, L. W. A complete protocol for in situ hybridization of messenger RNAs in brain and other tissues with radiolabeled single-stranded RNA probes. J. Histotechnol. 12, 169-181 (1989).

59. Lee, B. J. et al. TTF-1, a homeodomain gene required for diencephalic morphogenesis, is postnatally expressed in the neuroendocrine brain in a developmentally regulated and cell-specific fashion. Mol. Cell. Neurosci. 17, 107-126 (2001)

60. Son, Y. J. et al. TTF-1, a homeodomain-containing transcription factor, participates in the control of body fluid homeostasis by regulating angiotensiongen gene transcription in the rat subfornical organ. J. Clin. Invest. 278, 27043-27052 (2002).

61. Rage, F., Lee, B. J., Ma, Y. J. \& Ojeda, S. R. Estradiol enhances prostaglandin $\mathrm{E}_{2}$ receptor gene expression in luteinizing hormone-releasing hormone (LHRH) neurons and facilitates the LHRH response to $\mathrm{PGE}_{2}$ by activating a glia-to-neuron signaling pathway. J. Neurosci. 17, 9145-9156 (1997).

62. Watakabe, A., Komatsu, Y., Ohsawa, S. \& Yamamori, T. Fluorescent in situ hybridization technique for cell type identification and characterization in the central nervous system. Methods 52, 367-374 (2010).

63. Mastronardi, C. et al. Deletion of the Ttf1 gene in differentiated neurons disrupts female reproduction without impairing basal ganglia function. J. Neurosci. 26, 13167-13179 (2006).

64. Bilger, M., Heger, S., Brann, D. W., Paredes, A. \& Ojeda, S. R. A conditional, tetracycline-regulated increase in gamma amino butyric acid production near LHRH nerve terminals disrupts estrous cyclicity in the rat. Endocrinology 142, 2102-2114 (2001).

65. Berg-von der Emde, K. et al. Neurotrophins and the neuroendocrine brain: Different neurotrophins sustain anatomically and functionally segregated subsets of hypothalamic dopaminergic neurons. J. Neurosci. 15, 4223-4237 (1995).

66. Ramaswamy, S., Pohl, C. R., McNeilly, A. S., Winters, S. J. \& Plant, T. M. The time course of follicle-stimulating hormone suppression by recombinant human inhibin $\mathrm{A}$ in the adult male rhesus monkey (Macaca mulatta). Endocrinology 139, 3409-3415 (1998).

67. El, M. M., Ramaswamy, S., Sahu, A. \& Plant, T. M. Effects of orchidectomy on levels of the mRNAs encoding gonadotropin-releasing hormone and other hypothalamic peptides in the adult male rhesus monkey (Macaca mulatta). J. Neuroendocrinol. 12, 167-176 (2000).

68. Zar, J. H. Biostatistical Analysis 2nd edn (Prentice Hall, 1984).

69. Cukier, P. et al. Molecular and gene network analysis of thyroid transcription factor 1 (TTF1) and enhanced at puberty (EAP1) genes in patients with GnRH-dependent pubertal disorders. Horm. Res. Paediatr. 80, 257-266 (2013).

\section{Acknowledgements}

We thank Ms Maria Costa for performing the immunohistofluorescence studies. This work was supported by grants from NIH (5RO1 HD013254, U54 HD008610) to T.M.P., the US National Science Foundation (NSF: IOS1121691) to S.R.O., and a Marie Curie International Outgoing Fellowship within the seventh European Community Framework Programme (FP7-PEOPLE-2010-IOF) to J.M.C., C.A.T. and H.W. were supported by NIH Training grant T32 HD007133. H.W. was also supported by NIH Training grant T32 DK00768023. We also thank the staff of the Primate and Assay Cores of the Pittsburgh Specialized Cooperative Centers Program in Reproduction and Infertility for 
their help with conducting the experiments with male monkeys and for running the RIAs for monkey gonadotropins.

\section{Author contributions}

A.L., T.M.P. and S.R.O. designed the project and wrote the paper. A.L. was involved in all aspects of the study. S.R.O. coordinated the project and performed the intrahypothalamic injections of lentiviruses, FISH analysis and imaging studies. J.M.C. and C.A.T. measured mRNA by qPCR, run promoter assays and were involved in the rat in vivo experiments. V.M. extracted RNA for microarray analysis and measured mRNA by qPCR. H.W. performed in silico analysis of microarrays and gene co-expression network analysis. S.R. was involved in the studies using non-human primates including monkey surgery, blood sampling and radioimmunoassay of plasma hormone levels.

\section{Additional information}

Accession codes: The microarray data has been deposited into Gene Expression Omnibus hosted at the National Center for Biotechnical Information with the accession numbers GSE64396 and GSE64347.
Supplementary Information accompanies this paper at http://www.nature.com/ naturecommunications

Competing financial interests: The authors declare no competing financial interests.

Reprints and permission information is available online at http://npg.nature.com/ reprintsandpermissions/

How to cite this article: Lomniczi, A. et al. Epigenetic regulation of puberty via Zinc finger protein-mediated transcriptional repression. Nat. Commun. 6:10195 doi: 10.1038/ncomms10195 (2015).

(c) (i) This work is licensed under a Creative Commons Attribution 4.0 International License. The images or other third party material in this article are included in the article's Creative Commons license, unless indicated otherwise in the credit line; if the material is not included under the Creative Commons license, users will need to obtain permission from the license holder to reproduce the material. To view a copy of this license, visit http://creativecommons.org/licenses/by/4.0/ 